Portland State University

PDXScholar

$1-24-1994$

\title{
Lexico-Semantic Influence in Interlingual Transfer
}

Guy-Luc Levesque

Portland State University

Follow this and additional works at: https://pdxscholar.library.pdx.edu/open_access_etds

Part of the Bilingual, Multilingual, and Multicultural Education Commons Let us know how access to this document benefits you.

\section{Recommended Citation}

Levesque, Guy-Luc, "Lexico-Semantic Influence in Interlingual Transfer" (1994). Dissertations and Theses. Paper 4771.

https://doi.org/10.15760/etd.6655

This Thesis is brought to you for free and open access. It has been accepted for inclusion in Dissertations and Theses by an authorized administrator of PDXScholar. Please contact us if we can make this document more accessible: pdxscholar@pdx.edu. 


\section{THESIS APPROVAL}

The abstract and thesis of Guy-Luc Levesque for the Master of Art in Teaching English to Speakers of Other Languages were presented January 24, 1994, and accepted by the thesis committee and the department. COMMITTEE APPROVALS:
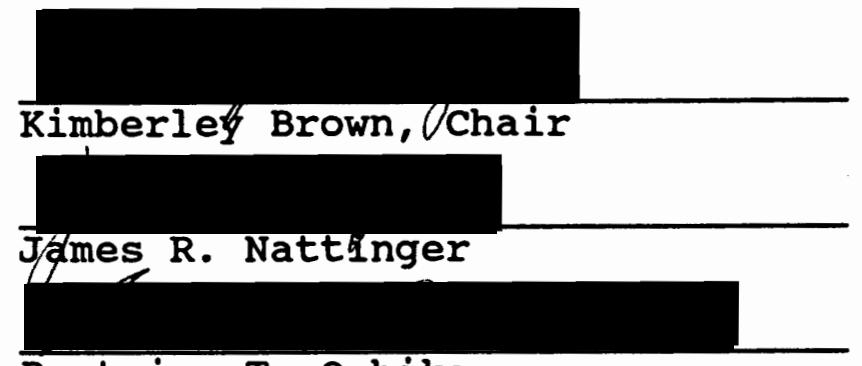
Beatrice T. Oshika

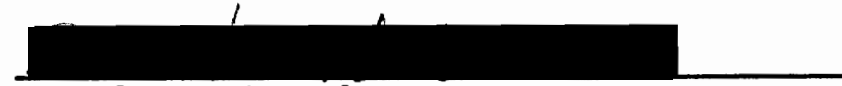
Suwako Watanabe Representative of the office of Graduate studies

DEPARTMENT APPROVAL:

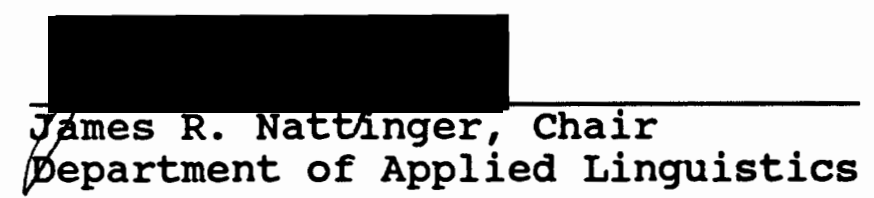

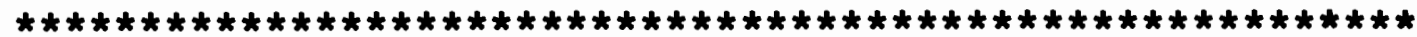

ACCEPTED FOR PORTLAND STATE UNIVERSITY BY THE LIBRARY

on

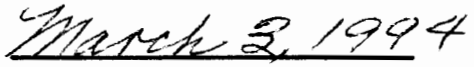




\section{ABSTRACT}

An abstract of the thesis of Guy-Luc Levesque for the Master of Art in Teaching English to Speakers of other Languages presented January 24, 1994, and accepted by the thesis committee and the department.

Title: Lexico-Semantic Influence in Interlingual Transfer.

The present study replicates research by Tomoko Takahashi (1984) on lexico-semantic patterns used by students in an acquisition poor environment. The purpose of the current study was to determine how an acquisition rich environment affects learners' use of four lexico-semantic patterns: congruence occurs when the Ll definition of a lexical item forms a one-to-one correspondence with the L2 lexical item; convergence occurs when the Ll lexical item has broader applications than the L2 lexical item; divergence occurs when the L2 lexical item has broader applications than the II lexical item; and semantic gap occurs when the II lexical item has no appropriate corresponding L2 lexical item (Takahashi, 1984).

The instrument, a lexico-semantics test, is the same instrument used in Takahashi's study. It was 
designed to measure which patterns are most frequently used by Japanese EFL students learning English.

The results, unlike Takahashi's, suggest that beginning and advanced ESL students use the four patterns equally well. No significant difference was found between the two groups. These results are contrary to what had been expected. However, they show that the proposed hierarchical order of difficulty of congruence, convergence, divergence and semantic gap is the same in both studies.

The results also indicate that the acquisition rich environment seems to dramatically improve beginners' performance of the four patterns. Since the instrument was designed for EFL students (an acquisition poor environment) it may not have fully challenged the advanced ESL students (an acquisition rich environment) while challenging the beginning students. This may have been due to the fact that the students in the present study received a great deal of input from the acquisition rich environment, which could account for their increased ability to restructure hypotheses about L2 vocabulary items.

In conclusion, more studies are needed to determine the complete role of the four lexico-semantic patterns in vocabulary acquisition. An expanded follow up study that fully tests the advanced and beginning ESI learners' ability could determine whether both groups 
progress along a language continuum with respect to the use of the four lexico-semantic patterns.

Furthermore, although the patterns may serve, in a limited capacity, as indicators of a learner's

difficulties in vocabulary acquisition, a wider body of research is needed before they can be applied in a language learning environment. 
LEXICO-SEMANTIC INFLUENCE

IN INTERLINGUAL TRANSFER

by

GUY-LUC ACHILLE LEVESQUE

A thesis submitted in partial fulfillment of the requirements for the degree of

MASTER OF ARTS

in

TEACHING ENGLISH TO SPEAKERS OF OTHER LANGUAGES

Portland State University

1994 


\section{DEDICATION}

This is dedicated to my parents, Janvier ét Genevieve Levesque. Since I am forever their child, any fruit that I bear is rightfully theirs; I would have it no other way. 


\section{ACKNOWLEDGEMENTS}

I am pleased to acknowledge the long time support of my parents and family. They have an undying faith in me that encourages me from one challenge to the next.

It is also a great pleasure to thank Dr. Kimberley Brown, my thesis adviser. She has given me sound advice throughout my thesis and studies at Portland state University.

This has been an arduous learning process and as any process of the kind, there are a great many people to thank. I would like to thank my study partner and dear friend Susan Merci, my friends Laurie Graham, Jim Freeman and Dominique sepcer. I would also like to thank my graduate committee. Their suggestions guided me through the final stages of the thesis.

Finally, special thanks to Kyung-Ah Chung, a true friend and a lifelong partner. She has supported me at every turn of this thesis, unselfishly leaving me to work when play would have been much more suitable to both of us. May I also be unselfish when her time comes. 
TABLE OF CONTENTS

PAGE

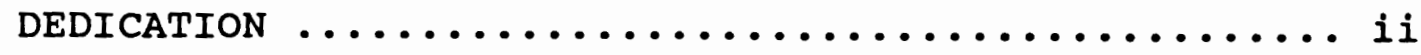

ACKNOWLEDGEMENTS $\ldots \ldots \ldots \ldots \ldots \ldots \ldots \ldots \ldots \ldots \ldots \ldots \ldots \ldots \ldots$

LIST OF TABLES $\ldots \ldots \ldots \ldots \ldots \ldots \ldots \ldots \ldots \ldots \ldots \ldots \ldots \ldots \ldots$

LIST OF FIGURES $\ldots \ldots \ldots \ldots \ldots \ldots \ldots \ldots \ldots \ldots \ldots$ ix

CHAPTER

I INTRODUCTION $\ldots \ldots \ldots \ldots \ldots \ldots \ldots \ldots \ldots \ldots$

II IITERATURE REVIEW $\ldots \ldots \ldots \ldots \ldots \ldots \ldots \ldots$.

PREDICTING AND ANALYZING ERRORS $\ldots \ldots \ldots \ldots 9$

Language Transfer and

Contrastive Analysis .............. 10

LEXICO-SEMANTICS IN L2 LEARNING $\ldots \ldots \ldots \ldots 16$

Semantically IIl-Formed Sentences .... 17

Translation-Transfer and

one-to-one Correspondence .........21

Translation and the

Lexico-Semantic Patterns .......... 24

Lexico-Semantic Patterns .........26

Hierarchical order

of the Four Patterns ............ 32

summary $\ldots \ldots \ldots \ldots \ldots \ldots \ldots \ldots \ldots$

III METHODOLOGY $\ldots \ldots \ldots \ldots \ldots \ldots \ldots \ldots \ldots \ldots \ldots$

Introduction $\ldots \ldots \ldots \ldots \ldots \ldots \ldots \ldots$ 


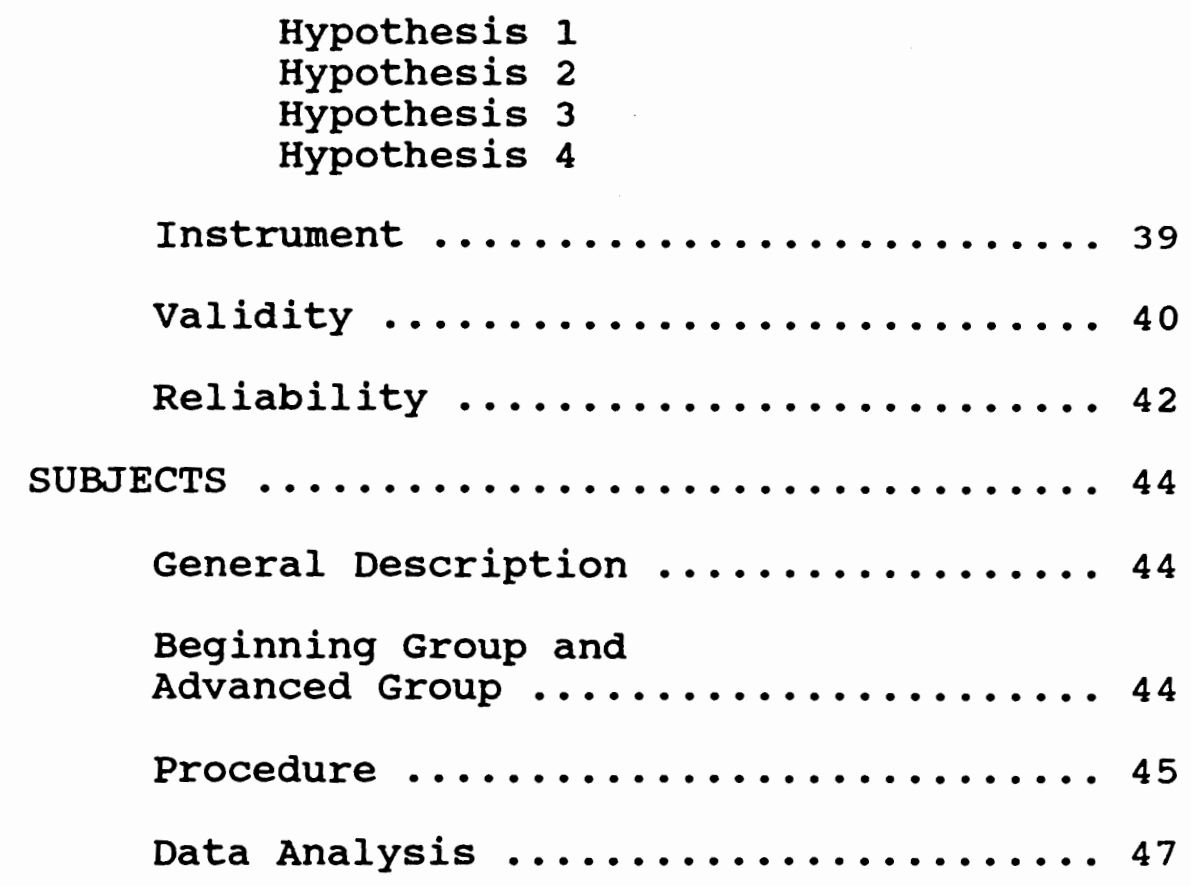

IV DATA ANALYSIS $\ldots \ldots \ldots \ldots \ldots \ldots \ldots \ldots \ldots$

Relative Difficulty of

the Four Patterns ............... 49

Verb Test Results

Adjective Test Results

SIGNIFICANCE OF THE FOUR PATTERNS ....... 54

Four Distinct Patterns ........... 54

Significant Difference

between the Two Groups ........... 56
Adjective Congruence
Adjective Convergence
Adjective Divergence
Adjective semantic Gap
Verb Congruence
Verb Convergence
Verb Divergence
Verb Semantic Gap

summary .................... 64

$V$ CONCLUSION, LIMITATIONS, SUGGESTIONS,

AND TEACHING APPLICATIONS ............ 65 
Conclusions and Limitations .......6 65

Suggestions ................. 74

Teaching Applications ........... 74

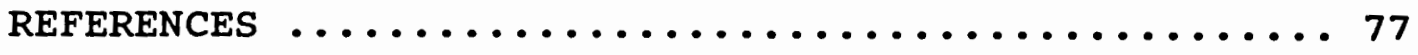

APPENDICES

A LEXICO-SEMANTICS TEST $\ldots \ldots \ldots \ldots \ldots \ldots \ldots 79$

B CONVERSION CHART $\ldots \ldots \ldots \ldots \ldots \ldots \ldots \ldots \ldots 8$ 
LIST OF TABLES

TABLE

PAGE

I Matrix of Spearman Correlation

Coefficients Beginning and

Advanced Adjective Test ............. 55

II Matrix of Spearman Correlation

Coefficients Beginning and

Advanced Verb Test ................ 56

III Adjective Congruence; a Comparison between the Beginning Group and the Advanced Group 57

IV Adjective Convergence; a Comparison between the Beginning Group and the Advanced Group 58

$\mathrm{V}$ Adjective Divergence; a Comparison between the Beginning Group and the Advanced Group 59

VI Adjective Semantic Gap: a Comparison between the Beginning Group

and the Advanced Group ................60

VII Verb Congruence: a Comparison between the Beginning Group and the Advanced Group .... 61

VIII Verb Convergence: a Comparison between the Beginning Group and the Advanced Group .... 62

IX Verb Divergence: a Comparison Between the Beginning Group and the Advanced Group ....66 63

$\mathrm{X}$ Verb Semantic Gap: a Comparison between the Beginning Group and the Advanced Group .... 63 


\section{LIST OF FIGURES}

FIGURE

PAGE

1. Average performance for the verb test

for the beginning group and

the advanced group ................ 50

2. Average performance for the adjective test

for the beginning group and

the advanced group .................. 52 
CHAPTER I

\section{INTRODUCTION}

The purpose of the current study is to replicate a study by Tomoko Takahashi (1984) in a different learning environment and compare the results from the two studies. This chapter begins with a description of Takahashi's study followed by an explanation of how the current study incorporates Takahashi's work.

Tomoko Takahashi conducted a study to determine whether Japanese EFL (English as Foreign Language) students use four lexico-semantic patterns as learning strategies. Her work focuses on the transfer of semantic values between a speaker's native language and his target language with respect to "content words", verbs and adjectives. The term "transfer" refers to learners who use semantic characteristics of their native language (NL) vocabulary and apply them to the target language (TL) vocabulary. Takahashi tested verbs and adjectives to determine if the results would be consistent with a previous study done by Shigenori Tanaka (1983). Tanaka focussed on the transfer of semantic values in respect to prepositions (locatives). Takahashi proposes that the four lexico-semantic 
patterns, congruence, convergence, divergence, and semantic gap are second language (L2) communication strategies which are used by learners to structure hypotheses about the TL vocabulary. She maintains that as learners progress, they restructure their hypotheses about the meanings of words, which are associated with the lexico-semantic patterns, and progressively commit fewer performance errors. Accordingly, because beginning learners have not progressed as far as advanced learners, they are expected to make more performance errors than advanced learners. Also, Takahashi suggests that the lexico-semantic patterns correspond to a learner's development depending on the level of the student and the difficulty of a pattern. Furthermore, Takahashi proposes that the lexicosemantic patterns have a predictable hierarchical order. She claims that the use of a pattern by any group corresponds with the hierarchical order. For example, beginning learners are expected to use all four lexicosemantic patterns in the same hierarchical order as advanced students, but they are not expected to use the patterns as successfully as the advanced students. In other words, all I2 learners will use an easy lexicosemantic pattern more successfully than a difficult lexico-semantic pattern. In addition, beginning learners will have a lower success rate throughout all 
four patterns in comparison to the advanced learners. That's because an easy lexico-semantic pattern, such as congruence, shows a one-to one correspondence between the native language and the target language whereas a difficult lexico-semantic pattern, such as semantic gap, shows no correspondence between the two languages. The lexico-semantic patterns will be explained in greater detail later in this chapter.

As mentioned, Takahashi's work is used as a model for the current study. In the present study, it is assumed that the four lexico-semantic patterns are used to structure hypotheses about the target language vocabulary. Also, it is assumed that a predictable hierarchical order exists for the lexico-semantic patterns and that the advanced group will perform the four patterns better than the beginning group. However, one fundamental difference between Takahashi's study and the present study is that Takahashi's study was conducted in an EFL environment, an acquisition poor environment. In an acquisition poor environment, learners do not benefit from the input of the target language and culture outside of the classroom. In contrast, the current study is conducted in an ESL (English as a second Language) environment--an acquisition rich environment. In an acquisition rich environment, students are immersed in the target 
language culture in the classroom as well as outside of the classroom; students benefit from the more regular and natural input of the target language and culture. Consequently, learners in an acquisition rich environment are expected to progress faster on a learning continuum because they are receiving more input.

The previous section briefly defined Takahashi's study and explained how the current study intends to make use of Takahashi's work. The next section will further define the four lexico-semantic patterns, describe in more detail the role of the patterns in the current study, and state the hypotheses in this study.

Takahashi defines the lexico-semantic patterns as follows: 1) Congruence occurs when the L1 definition of a lexical item forms a one-to-one correspondence with the L2 lexical item (Takahashi, p.89, 1984); 2) Convergence occurs when the Ll definition of a lexical item has broader applications than the L2 lexical item (p.84); 3) Divergence occurs when the L2 lexical item has broader applications than the Ll lexical item. In other words, it is the counterpart to convergence (p.89); 4) Semantic gap occurs when the L1 lexical item has no appropriate corresponding word in the second language (p.169). Semantic gap, in other words, occurs when the L1 lexical item has no equivalent L2 lexical 
item. The four patterns can be illustrated as follows:

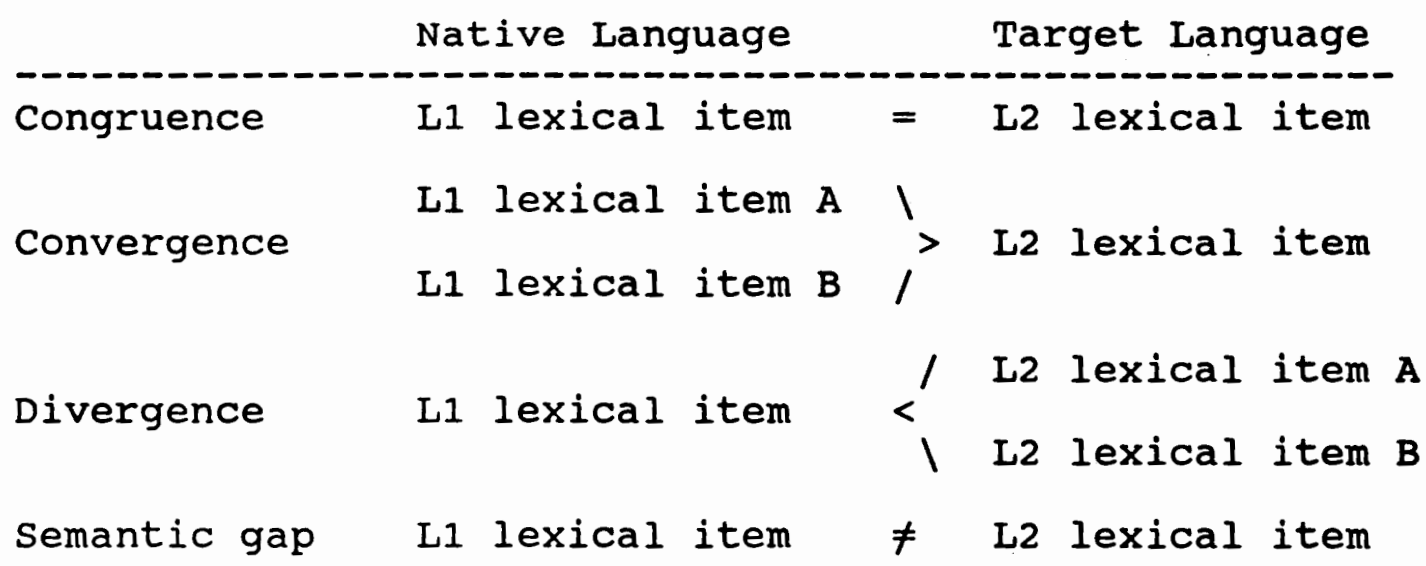

Takahashi proposes that lower level students rely more on congruence as a language learning pattern than do advanced students. The beginning students' relatively heavy reliance on one-to-one correspondence of words represents an equational framework which can lead to overgeneralization, which in turn, can lead to performance errors. Advanced students, on the other hand, rely less on the assumption that there is a oneto-one correspondence between NL items and TL items. Rather, they use more appropriate learning strategies such as convergence, divergence, and semantic gap. Therefore, advanced learners will typically have far fewer performance errors than their novice counterparts. This was evident in Takahashi's study, and similar results are expected in the present study.

As previously mentioned, Takahashi's research builds on a study conducted by Tanaka (1983). Tanaka 
asks whether Japanese beginning L2 learners rely more on their native language (NL) than Japanese advanced L2 learners. Tanaka concludes that the LI vocabulary serves as a grid, a basis, from which L2 learners initially build hypotheses about the TL. Learners restructure their hypotheses on a continuum that gradually relies less on a one-to-one equivalence of word meanings, i.e., congruence, and more on other lexico-semantic strategies appropriate to L2. The current study accepts Tanaka's conclusion. Consequently, three hypotheses are proposed in the current study to address Tanaka's conclusion:

Hypothesis 1: There is a hierarchy of difficulty to perform the four lexico-semantic patterns. On a scale from least difficult to most difficult, the patterns are congruence, convergence, divergence, and semantic gap.

Hypothesis 2: Beginning learners will demonstrate a higher frequency of lexico-semantic errors on the semantics test when the lexico-semantic patterns of convergence, divergence and semantic gap apply. This is due to the beginning learner's reliance on congruence, an equational framework where $\mathrm{L} 1=\mathrm{L} 2$, as a communication strategy. 
lower frequency of lexico-semantic errors on the semantics test when the lexico-semantic patterns of convergence, divergence, and semantic gap apply.

In addition, a fourth hypothesis is proposed for the current study to address the notion that although native Japanese students learning English will perform differently in an acquisition poor environment compared to an acquisition rich environment, the hierarchical order of the patterns will remain the same.

Hypothesis 4: The hierarchical order of the four lexico-semantic patterns will be the same in an acquisition poor environment as in an acquisition rich environment.

If true, these hypotheses may support teachers' use of the four lexico-semantic patterns as indicators of a learner's developmental progress in vocabulary acquisition. Additionally, the four lexico-semantic patterns may help teachers to better understand the difficulties that $L 2$ learners encounter in learning new vocabulary.

In conclusion, it is the intent of the present study to determine whether beginning level Japanese ESL students use certain lexico-semantic patterns more often than advanced level Japanese ESL students, whether the four lexico-semantic patterns occur in a predictable 
hierarchical order, and whether a learner's use of the lexico-semantic patterns is affected by an acquisition rich environment in comparison to an acquisition poor environment.

Chapter II presents a summary of the relevant research. Chapter III outlines the research method followed in this study. Chapter IV describes the results from the data. Chapter $V$ is a conclusion in which the results of the data are discussed and recommendations for future research made. 
CHAPTER II

\section{LITERATURE REVIEW}

Since the current study is based on the work by Tanaka and Takahashi, it is useful to review their theoretical bases and definitions. other authors' work are examined as it pertains to the subject.

The first section of the literature review, Predicting and Explaining Errors, gives a brief history of contrastive analysis, explains the application of lexico-semantic errors, and discusses the general function of language transfer in the scope of the current study.

\section{PREDICTING AND ANALYZING ERRORS}

The strong and the weak version of contrastive analysis are used to predict and analyze data in the present study, although, as will be seen, a compromised version of contrastive analysis will be adopted because these two theories alone are not sufficient to account for all the data. The strong and the weak versions are explained by defining them in terms of positive and negative transfer and other related notions. 
Lanquage Transfer and Contrastive Analysis

Most language experts agree that a speaker's native language (NL) influences the acquisition of a target language (TL) (Schachter, 1974; Gass, 1988; Corrale, 1989). Furthermore, as seen in the present study, the influence of a speaker's native language can sometimes be realized as language transfer. Tanaka and Abe (1984), for example, propose that language transfer occurs when L2 learners utilize elements of their first language when approaching the task of learning another language. For the purpose of the current study, positive transfer is when a learner transfers NL elements to the TL that are shared by the TL, and no error occurs. Negative transfer is when a learner transfers NL elements to the TL that are not shared by the TL, and an error does occur. To understand the role of language transfer, and how it is viewed in the current study, we must first consider contrastive analysis since it is used as a tool in the current study for predicting and analyzing the data.

Essentially, there are two versions of contrastive analysis: the strong version, also known as Contrastive Analysis; and the weak version, also known as Error Analysis. To avoid confusion, this study refers to the two versions as the strong version and the weak version. When the term "contrastive analysis" is used, it refers 
to the combined theories of the weak version and the strong version unless otherwise noted. As Takahashi (1984) pointed out, there are drawbacks to both the strong and the weak versions and neither alone can account for the results of her study. She proposed a combination of the two versions to address the problem. As it will be seen, Takahashi's proposal seems justified in the current study as well.

The strong version predicts that the primary cause of difficulty in L2 learning is interference from previous linguistic knowledge from the learner's NL and is exemplified in the works of Lado (1957) and Stockwell (1968), among others. This theory rests on the notion that interference represents negative transfer, which is caused by differences between the native language and the target language, and that these differences can be determined by comparing the two languages. Jakobovits (1969) suggests that the greater the difference between two languages the greater the difficulty learners will have to learn the TL. This suggests that errors can be predicted by comparing differences in the TL with those in the NL.

Based on differences between the NL and the TL, the four lexico-semantic patterns can be explained in terms of frequency of errors and differences between the patterns. It is predicted that fewer errors will occur 
with congruence (CG) lexical items than items of other lexical patterns because congruence shows little of no difference between the NL and the TL. With congruence, the potential for positive transfer far outweighs negative transfer. More errors will occur with convergence (CV) lexical items than CG lexical items because $\mathrm{CV}$ items have more differences between the NL and the TL than CG items. With convergence, there is more potential for positive transfer than negative transfer, but the difference is not considerable; more errors will occur with divergence (DV) lexical items than CV lexical items because DV items have more differences between the NL and the TL than CV items. With divergence, there is more potential for negative transfer than positive transfer, but the difference is not considerable; more errors will occur with semantic gap (SG) lexical items than DV lexical items because SG items have more differences between the NL and the TL than DV items. With semantic gap, the potential for negative transfer far outweighs that for positive transfer. A scale predicting the occurrence of errors of the four patterns would be like this: CG items are easier to perform than $\mathrm{CV}$ items; $\mathrm{CV}$ items are easier to perform than DV items; and DV items are easier to perform than SG items. In the instances above, negative transfer is said to impede learning and positive 
transfer is said to facilitate learning.

A problem with the strong version is that its focus on predicting errors emphasizes negative aspects of the language and overlooks the usefulness of errors as a diagnostic tool. For example, errors need not be considered something a learner needs to avoid, but rather, could be considered as an indication of a learner's progress, which in turn, could be useful to teachers in curriculum design. Using a theory which treats most transference as negative is thus somewhat limiting.

In the late 1960's, some linguists began to express their dissatisfaction with the predictive role claimed by the strong version because of its overemphasis on error prediction (i.e., Catford, 1968; Lee, 1968). By the early 1970's, the strong version was essentially replaced by the weak version. The weak version analyzes, rather than predicts, observable learner errors, and perhaps most importantly, it views errors as a natural progression in the developmental process of language learning that can reveal useful information about L2 development. However, while a useful tool, the weak version alone does not completely account for the role of transfer in language learning and, consequently, it is unable to fully account for student responses on the lexico-semantics test. 
The instrument used in the current study predicts that learners use the four lexico-semantic patterns according to the proposed hypotheses. If the students' responses are not in accord with the predicted answers, the learner errors must then be analyzed. This requires the use of both versions: the strong version to predict errors and the weak version of to account for them. In addition, Schachter's position is that the strong version should be combined with the weak version to account for another factor: hard-to-observe errors such as an avoidance strategy. In a study of relative clause errors, which included Iranian, Arab, Chinese, and Japanese learners of English, Schachter found that Chinese and Japanese students demonstrated fewer errors when using relative clauses at a time when more relative clause errors were predicted. Schachter surmised that the Chinese and Japanese students avoided the use of relative clauses, which explained why they made fewer errors. She concluded that the weak version did not account for such strategies as avoidance, which can mask errors that students produce.

Combining the strong and weak version of $C A$ fulfills the need for predicting and interpreting language transfer. However, the strong and the weak version are only concerned with the influence of transfer from the NL to the TL. 
In later research, Schachter (1981) proposes that learners are not only influenced by their native language, but by their target language as well. Accordingly, to more accurately predict and interpret language transfer, the influence of the TL on the NL must also be considered.

The concept of a universe is not that of a static list of hypotheses, but rather one which expands as the learner gains experience. That is, as the learner becomes more proficient in the target language, he will develop hypotheses on the basis of experience with the target, and these will be added to the ever-expanding list of hypotheses available. (Schachter, 1981, from Takahashi, p.18, 1984)

Schachter's statement strongly suggests that the more experience learners have in using L2, the more hypotheses they will form about the TL. Furthermore, the hypotheses are not only influenced by the NL, but by the TL as well. This suggests that the strong version and the weak version are not sufficient to account for the influence of the TL. Schachter's statement also suggests that learners start out with a small list of hypotheses that grows as the learner experiences more of the TL. This indicates that beginning level L2 learners form hypotheses about the TL that are restructured as the learners gain experience and thereby progress on a learning continuum.

These notions are important to the current study for three reasons: (1) the two groups used in this study 
are distinguished by level of proficiency; (2) this study hypothesizes that beginning students rely more on one kind of language learning pattern (congruence) than do advanced students; and (3) the current study is conducted in an acquisition rich environment where students have a lot of data on which to restructure their hypotheses about the TL.

In conclusion, four notions are proposed for the current study: (1) I2 transfer errors are predictable; (2) I2 transfer errors, positive and negative, can be interpreted to reflect a learner's progression along an L2 learning continuum; (3) a learner's L2 transfer is not only influenced by their NL, but by the target language as well; and (4) the successful use of a pattern of transfer depends on a learner's I2 proficiency.

\section{LEXICO-SEMANTICS IN L2 LEARNING}

This section introduces key concepts for understanding the development and purpose of the present study. It defines: semantically ill-formed sentences to discern their function in the lexico-semantics test; collocational restrictions and the role they play in determining semantic anomalies; translation-transfer and how it is used as an L2 strategy; and transfer and its correlation to the lexico-semantic patterns. 
Furthermore, the lexico-semantic patterns are clearly defined, and the hierarchical order of the four patterns is explained in relation to positive and negative transfer.

\section{Semantically Ill-Formed Sentences}

Semantically ill-formed sentences involve the substitution of linguistic items which cause a difference in meaning in structurally well-formed sentences. They occur when L2 learners translate words or phrases from their native language into the target language, but the translation does not convey the same meaning as in the native language. For example: "The world has become narrow", instead of "The world has become smal1."

In the above example, Takahashi (1984) proposes that this type of deviation (choosing narrow instead of smal1) is due to an inappropriate choice of words, and that this choice can sometimes be accounted for by direct translation of Japanese expressions into English. If this is so, then how does a learner come to make that choice? Tanaka (1983) and Takahashi (1984) suggest that Japanese learners make this choice because they perceive an equational framework between the Japanese word and the English word. In other words, speakers assume that the meaning of a translated word conveys the same meaning in the target language (English) as in the 
native language (Japanese), while in fact, it conveys a different meaning than was intended.

For example, "the Japanese adjective /semai/ can mean both "narrow" and "small" in Japanese, i.e., /semai michi/ (narrow path) and /semai heya/ (small room)." (Takahashi, p.29, 1984). Accordingly, Takahashi proposes that Japanese learners of English will use "narrow" and "small" interchangeably in English as they do in Japanese, irrespective of the restrictions of the context. That is, the learner does not recognize any semantic (meaning) difference between the two words; the results in Takahashi's study indicate this is especially true for beginning learners. As would be expected, this leads to lexico-semantic errors. It can be said, therefore, that the learner's use of direct translation (equational framework) of Japanese expressions increases the probability of transfer errors in the TL, which is especially true for beginning learners.

Moreover, semantic transfer errors occur because learners do not have a clear understanding of the collocational restrictions for a particular TL expression. Collocational restrictions reflect the limits imposed on words by a language and how such limits are unsuspectingly violated by unsuspecting L2 learners. These rules are violated when the items in a sentence conform to grammatical rules, yet show some 
"strangeness or unintelligibility" associated with meaning.

For example, in the sentence "The world has become small", the English word small collocates with world. In this context, narrow does not collocate with world. However, a native Japanese student using direct translation as a strategy may overlook the differences in the collational restrictions between Japanese and English, which in turn may lead to miscommunication (Takahashi, p.30, 1984). The collocational restrictions placed on a word can more accurately be explained in terms of the semantic features of a word which limit the environment in which a word can be applied. When transfer occurs, L2 learners transfer the NL semantic features to a TL word that they assume corresponds with the NL word. For example:

$$
\begin{array}{ll}
\text { narrow } & \text { [+width, -maximal] } \\
\text { small } & \text { [+size, -maximal] } \\
\text { /semai/ } & \text { [+space, twidth, tsize, -maximal] } \\
\text { /chiisai/ } & \text { [+size, -maximal] } \\
\text { The features of the English word narrow are }
\end{array}
$$
described as [+width, -maximal], while the word small has the features [+size, -maximal]. In comparison, the Japanese word /semai/ is described as having [+space, 士width, \pm size, -maximal], and the Japanese word /chiisai/ has the features [+size, -maximal]. The features for /chiisai/ are the same as those of small. 
If a Japanese learner uses a one-to-one correspondence between the Japanese and English items, then the I2 learner is expected to associate small with /chiisai/. While this type of transfer is a simple one-to-one correspondence, it overlooks more complex relationships of words between the NL and the TL.

In a later section, we shall see how Takahashi uses collocational restrictions to create the lexicosemantics test sentences. While collocational restrictions are not brought to the attention of the subjects in the present study for any data gathering purposes, they are necessary to explain the relationship of the cue words with respective sentences.

The present study predicts that the extent of transfer errors due to the violation of collocational restrictions is relevant to the student's level of proficiency: a lower level student will demonstrate more errors than a higher level student. This is because a lower level student is more likely to generalize a oneto-one correspondence between their NL and the TL. This type of generalization overlooks more complex relationships between NL words and TL and, therefore, can lead to semantically ill-formed sentences.

Furthermore, although the current study does not test for semantic features and collocational restrictions, it does predict that some performance 
errors occur because words or phrases are used by a speaker in violation of the target language's collocational restrictions. The language learners' assumptions of the collocational restrictions of a word or phrase are influenced by language transfer. When language learners violate the collocational restrictions, they produce semantically ill-formed sentences.

Translation-Transfer and one-To-one correspondence

Translation-transfer is a concept which helps to explain why L2 learners rely on a one-to-one correspondence and how learners progress by restructuring their hypotheses about TL words, which can ultimately lead to successful acquisition of TL items. This helps to explain the process in which beginning learners make more errors than advanced learners.

Takahashi and Tanaka suggest that the "translationtransfer phenomenon" is a fundamental L2 strategy used by students to learn L2 vocabulary. The translationtransfer phenomenon involves two distinct yet closely related processes: first, the establishment of analogies between NL and TL expressions or the association of NL and TL words; and second, the manifestation of such analogies and association in TL production (Takahashi, p.36, 1984). The first notion refers to idea that learners begin by forming hypotheses about the TL using 
whatever knowledge they already have, which includes their NL. The second notion refers to the idea that learners must somehow test their hypotheses and that this is done by using the TL. This accounts for part of the process students undergo in learning a second language.

Tanaka (pp.25-33, 1983) provides insight into translation-transfer as part of L2 learning. Terming it the "search-translation equivalence strategy," he proposes that when L2 learners are learning the meaning of new TL items, they may search for a "translation equivalence" which has a one-to-one correspondence with their own NL. This initial stage of learning is characterized by the general assumption of equational frameworks. As learners advance in language learning, however, they become aware that the one-to-one correspondence does not always hold true. When this happens, L2 learners have to restructure the internalized framework to accommodate new knowledge (Tanaka, 1983; Takahashi, 1984). Restructuring facilitates learning and allows L2 learners to reform hypotheses about the TL item. The process of restructuring is necessary for learners to progress on an L2 learning continuum.

Takahashi and Tanaka indicate that translation as a communication strategy triggers the restructuring 
process. Takahashi proposes that this occurs because "the use of a communication strategy provides the learner with an opportunity to apply the one-to-one correspondence and thereby test its validity in context" (Takahashi, p.38, 1984). The feedback a learner receives serves as an indication as to whether restructuring needs to continue. Furthermore, the more opportunity learners have to restructure their hypotheses, the faster they will progress along an L2 learning continuum.

The subjects in the present study live in an acquisition rich environment. As proposed earlier, an acquisition rich environment should provide more natural input from the TL than a restricted environment. It is likely, therefore, that the subjects in the current study had more opportunities to restructure their hypotheses than the subjects in the Takahashi study.

In conclusion, as indicated in this section, translation-transfer as a communication strategy is an important step that leads to restructuring, which in turn is necessary for a learner's progress. The amount of restructuring a learner has undergone can indicate a learners developmental level. Therefore, learning environment may be an important factor in L2 learning. The difference in environments between the present study and Takahashi's study will be considered in determining 
how a rich environment affects the lexico-semantic performance of L2 learners.

\section{Translation and the Lexico-semantic Patterns}

The concept of translation-transfer indicates that one possible source for semantic transfer is translation and that translation, as a communication strategy, triggers restructuring if there is feedback. Tarone (1983) proposes that direct (literal) translation is a realization of transfer, and is used as a communication strategy. Direct translation occurs when learners translate word for word from the native language, i.e., "He invites him to drink" instead of "They toast one another" (Tarone, from Gass, 1988). In this example, the learner substituted the phrase "He invites him to drink" for "They toast one another" where the former refers to a phrase from the speaker's native language; this example of direct translation reflects learners' use of previous knowledge to communicate a message.

Takahashi agrees with Tarone and associates the notion of direct translation to the lexico-semantic patterns. Takahashi suggests that a learner frames his intended message (M1) in the $\mathrm{NL}$ first and then translates it into the TL (M2). Accordingly, the message conveyed (M2) may or may not be semantically equivalent to the intended message (M1). This semantic relationship can be represented in three ways: 
1) M1 is semantically equal or very closely corresponds to M2.

2) M1 only partially corresponds semantically to M2.

3) M1 does not correspond semantically to M2.

These three possibilities correspond to the three semantic patterns used in Tanaka's study (1983): congruence, split, and semantic gap. In addition to these three patterns proposed by Tanaka, Takahashi chooses to redefine Tanaka's split pattern as divergence and add congruence as a fourth lexico-semantic pattern. Tanaka did not include the convergence pattern in his study because Japanese prepositions do not show any clear correspondence to the convergence pattern. Convergence occurs when a TL item converges into several NL items. However, since Takahashi is concerned with adjectives and verbs in her study where convergence does occur, she had to include this type of correspondence between the two languages.

The above three possibilities are thus expanded to four and can be more technically defined as follows: 1) Congruence occurs when the L1 definition of a lexical item forms a one-to-one correspondence with the L2 lexical item (Takahashi, p.89, 1984).

2) Convergence occurs when the L1 definition of a lexical item has a broader application than the L2 lexical item (p.84). 
3) Divergence occurs when the L2 definition of a lexical item has a broader application than the $\mathrm{L} 1$ lexical item (p.89); divergence is the counterpart to convergence. 4) Semantic gap occurs when the L1 lexical item has very little or no appropriate corresponding word in respect to the L2 lexical item (p.169).

These definitions serve to clarify the four patterns in terms of the NL and the TL. However, the patterns require further definition for the current study. This is done in the following section.

\section{Lexico-Semantic Patterns}

The current study uses Takahashi's instrument to measure the use of the lexico-semantic patterns among native Japanese learning English. It is, therefore, important to understand Takahashi's process in creating the lexico-semantics test and in choosing the set of function words (adjectives and verbs) used in the test.

In addition, the four lexico-semantics patterns are further defined in relation to one-to-one correspondence (an equational framework) and the importance of restructuring is reviewed to give a clear understanding of the patterns' function in the current study.

The concept of translation-transfer as a communication strategy and its realization in direct transfer suggest that learners will make errors that can be explained in terms of lexico-semantic patterns. 
These patterns may account for instances such as the interchangeable use of "small" and "narrow" by beginning Japanese L2 learners of English.

only two researchers have extensively considered the significance of lexico-semantic patterns in language transfer: Takahashi and Tanaka. As mentioned, Tanaka (1983) considered lexico-semantic patterns pertaining to the correspondence of prepositions (or locatives) between the TL and the NL in an acquisition poor environment. Takahashi (1984) expanded on Tanaka's study by focusing on the correspondence of lexicosemantic patterns pertaining to verbs and adjectives in the same environment.

To examine whether the patterns proposed by Tanaka also hold true with content words (verbs and adjectives), Takahashi crewt:od an instrument to test Japanese learners' use of lexico-semantic patterns with respect to adjectives and verbs; it is not clear why Takahashi chose adjectives and verbs as opposed to other content words (i.e., nouns) except that she wanted to expand on Tanaka's study on prejositions by investigating other linguistic categories.

Takahashi conducted two preliminary tests to disclose the type of translation patterns (lexicosemantic patterns) used by native Japanese speakers when learning English vocabulary and to establish a set of 
words that could be used in the instrument.

In Takahashi's first preliminary study, twenty Japanese graduate students studying in New York were each asked to give Japanese equivalents to selected English verbs and adjectives in a null context. She chose verbs and adjectives ("cue words") from a study conducted by Thorndike (1932) on the first 500-1,000 most frequently used words by native speakers of English. The words were also correlated with frequently taught vocabulary in the Japanese school system to assure that there would be a strong chance of the words being familiar to the students. The responses of the first test were then used in the second test. Takahashi's second preliminary study established the full relationship of the cue words in which the Japanese words given as responses in the first study were then used as cue words to elicit responses in English. The test showed the semantic correspondence between the English words and the Japanese words. For instance, in some situations, such as with the words "look, see", and "watch", there are three distinct words in English which are used in different contexts with different Japanese collocational restrictions. However in Japanese, the collective meaning of "look, see", and "watch" is equal to one word "/miru/" with the same collocational restrictions. This shows a clear case of divergence. 


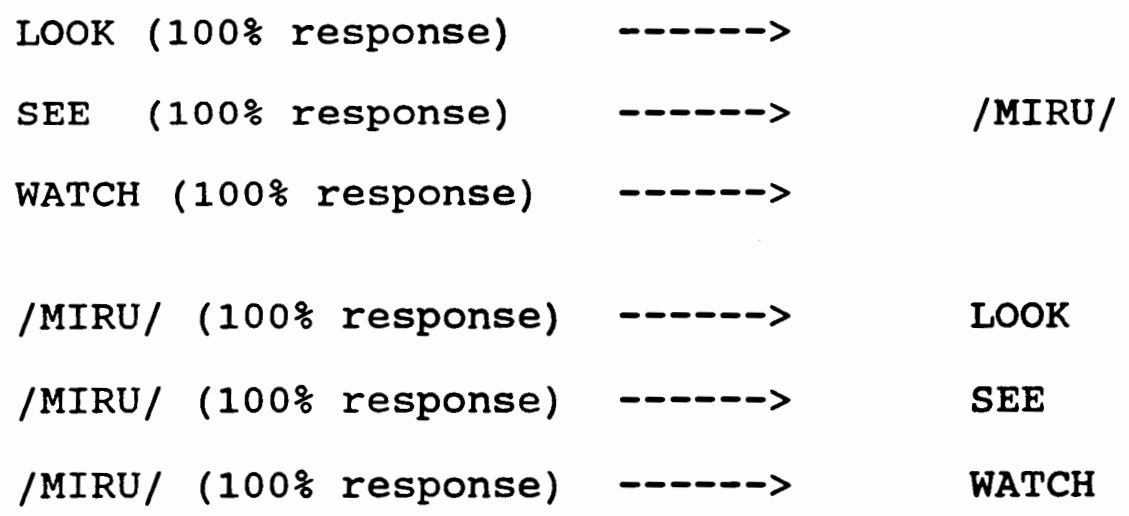

Takahashi set a significance level at $85 \%$; that is, when the English vocabulary words "look, see", and "watch" elicited the response /miru/ over $85 \%$ of the time as the Japanese equivalent and when the same Japanese word corresponded to each of several English words $85 \%$ or better, it was concluded that the correspondence pattern between the NL and the TL is divergence (Takahashi, 1984).

The analysis of the data from the two preliminary studies conclusively established the relationship of the words in terms of the four lexico-semantic patterns-congruence, convergence, divergence and semantic gap, which formed the basis for the lexico-semantics test used as the instrument for the present study. Once the patterns were established, a lexico-semantics test was created to measure L2 learners' use of the four patterns and account for the semantic relationship of the cue words and the sentence. Furthermore, she had to account for a learner's choice of word in a sentence.

In order to accomplish this, Takahashi (p.84, 1984) 
describes the restrictions on the use of words by means of "collocational relations allied with semantic features". For example, while /yuderu/ and /wakasu/ are both considered to have the features [+water] and [theat], /yuderu/ takes as its direct object a noun phrase marked with the feature [+food]. In comparison, the use of /yuderu/ is motivated by the feature [+food] while the use of /wakasu/ is motivated by the feature [+water] (see example below). The "lexical motivator" of the translated Japanese word is believed to play a role in terms of which word a student chooses to associate as the proper English translation.

The relationship of /yuderu/ and /wakasu/ corresponding to the English word boil, can be illustrated as follows (Takahashi, p.85, 1984):

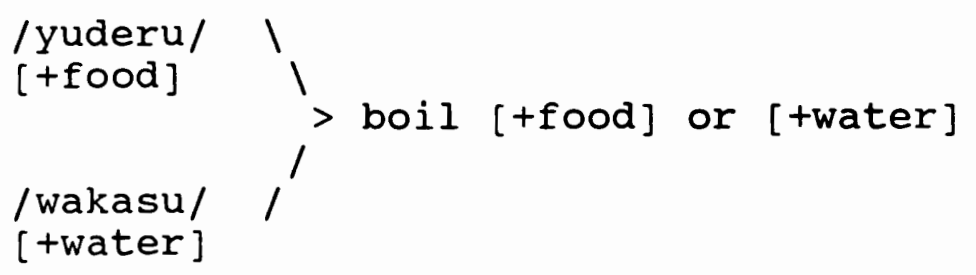

Regarding the above example, Japanese learners may be aware of a difference between the items in the $\mathrm{NL}$, but they may not be aware that these items are coalesced into one item in the TL (a convergence pattern). The acquisition of an item represented by the convergence pattern, therefore, requires learners to blend the NL items into one TL item. This coalescing process, in terms of congruence, is called "blending". 
On the other hand, as noted earlier, divergence is the counterpart of convergence; it operates on the same principle as convergence, only from the reverse standpoint. The learner in this circumstance faces a situation where several or more items in the TL correspond with one item in the NL. In this case, the coalescing process is called "sifting".

Semantic gap, the fourth lexico-semantic pattern, is the one-to-one relationship that a learner believes to exist between a NL item and a TL item, while in actuality, no direct correlation can be drawn between the NL item, the intended meaning, and the proposed TL item. This relationship can be illustrated as follows: in semai <seken>, the learner cannot depend on the congruence pattern (/semai/ = narrow) because the phrase narrow world will be judged semantically different from the learner's intended meaning small world. The application of the one-to-one correspondence, congruence, perceived by the learner fails in this instance, due to a semantic gap between the languages, where this correspondence does not exist.

In sum, "restructuring" of the initial one-to-one equational framework is required in order for the learner to achieve some measure of success where the semantic patterns convergence, divergence and semantic gap apply (Takahashi, 1984 and Tanaka, 1983). For 
example, although a learners may initially perceive "narrow" and "small" to be equal (an equational framework), they will eventually learn that "narrow" does not equal "small" in all linguistic environments. Such instances may cause learners to restructure their hypotheses about TL items and, accordingly, progress along an L2 learning continuum until they achieve their language goals.

\section{Hierarchical Order of the Four Patterns}

The four lexico-semantic patterns have just been defined and explained in terms of English and Japanese lexico-semantic correspondence. In addition to this, the four lexico-semantic patterns are assumed to have a predictable hierarchical order (Takahashi, 1984). To understand how a hierarchical order is concluded, we must consider the four lexico-semantic patterns and their relation to positive and negative transfer. Furthermore, the four patterns' relation to positive and negative transfer will help define the level of difficulty of the lexico-semantic patterns. We will conclude that the hierarchical order of the four patterns, based on their difficulty to perform, is congruence, convergence, divergence, and semantic gap.

We briefly discussed negative and positive transfer in the first section of the current study. We concluded that negative transfer impedes and positive transfer 
facilitates L2 learning. These assumptions were based on Tarone's notion that difference equals or causes difficulty. Associating positive and negative transfer with the four lexico-semantic patters is a relatively simple association. The lexico-semantic features that are shared between a NL item and a TL item will result in positive transfer. The lexico-semantic features that are not shared between a NL item and a TL item can result in negative transfer. To put this in terms of the lexico-semantic patterns, congruence predicts that the NL item and the TL item share semantic features; convergence predicts that the NL item and the TL item share fewer semantic features in comparison to congruence; divergence predicts that the NL item and the TL item share fewer semantic features in comparison to convergence; and semantic gap predicts that the NL item and the TL item share very few or no semantic features. Although this illustrates the relationship of the four lexico-semantic patterns in terms of shared features with positive and negative transfer, the lexico-semantic patterns must also be explained in terms of one-to-one correspondence, blending, sifting, and complete restructuring. These four elements help to explain the full reason for the patterns difficulty to perform. of the four patterns, it is assumed that congruence is easier to perform than the other three lexico- 
semantic patterns because it is related to one-to-one correspondence and positive transfer. When one-to-one correspondence is assumed and congruence between the NL and the TL exists, no errors occur and positive transfer takes place (Takahashi, p.90, 1984). Accordingly, positive transfer facilitates learning.

Convergence, divergence and semantic gap, on the other hand, are associated, in part or in whole, with negative transfer. In other words, although congruence may be assumed by the learner, semantic differences may exist between the corresponding NL and TL words which can cause performance errors (negative transfer). of these three patterns, convergence poses the least difficulty because it generally requires learners simply to overlook distinctions to which they have grown accustomed (Brown, p.152, 1980). Takahashi suggests that overlooking a distinction in the process of blending is easier than forming new distinctions because it essentially does not require the learner to learn anything new (Takahashi, 1984). Accordingly, the pattern convergence is likely to cause less negative transfer than divergence or semantic gap.

In contrast, however, partial restructuring is required for divergence and completely new restructuring is required for semantic gap. When comparing divergence and semantic gap, Takahashi posits that divergence is 
generally less difficult. This means that divergence causes less negative transfer than semantic gap because of fewer differences between NL items and TL items. This is based on the notion that divergence already has all the essential elements present, so the learner primarily maintains the L1=L2 framework and adjusts additional semantic items to allow for the new context. Because this involves less restructuring than semantic gap, we can conclude that fewer occurrences of negative transfer are likely to occur.

on the other hand, if an item is rejected due to the condition of semantic gap, the learner has to go outside the $L 1=L 2$ framework to restructure the rejected item. Each occurrence of restructuring is triggered by negative transfer. In an acquisition rich environment, there are, of course, more opportunities for feedback. It is generally viewed, therefore, that semantic gap is more difficult than divergence.

Based on these notions about the lexico-semantic patterns, Tanaka (1983) and Takahashi (1984) propose that the learners' difficulty to perform the lexicosemantic patterns indicates a hierarchical order.

The hierarchical order and lexico-semantic patterns proposed by Tanaka is congruence > split > and semantic gap. However, as mentioned earlier, Takahashi renames the split pattern divergence and adds its counterpart, 
convergence, to account for the type of transfer that occurs with content words. Because the current study, like Takahashi's study, is concerned with content words, Takahashi's four lexico-semantic patterns and their hierarchical order are adopted as the standard for the present study. The hierarchical order of the four lexico-semantic patterns from least difficult to most difficult is congruence > convergence > divergence > semantic gap. The present study will determine if this order holds true in an acquisition rich environment.

\section{Summary}

In conclusion, the four patterns of congruence, convergence, divergence, and semantic gap have been defined in terms of one-to-one correspondence, blending, sifting, and complete restructuring. Strategies such as congruence, convergence, divergence, and semantic gap are shown to be used when learning new vocabulary. Such strategies, however, are not always successful. It was also shown how negative transfer, which can result in errors, may occur because the correspondence of a lexical item in the TL does not correspond in part or in whole to the lexical item in the NL. This study aspires to understand the lexical relationship between NL items and TL items and their influence on vocabulary performance and to determine if Takahashi's results hold true in an acquisition rich environment. 
CHAPTER III

\section{METHODOLOGY}

\section{Introduction}

The current study is concerned with lexico-semantic transfer in an acquisition rich environment. In an acquisition rich environment students are immersed in the target language culture and benefit significantly from the input of the target language culture (Krashen, 1989), unlike those in an acquisition poor environment. As mentioned earlier, the Takahashi study was concerned with native Japanese students learning English as a second language in an acquisition poor environment, Japan. This fundamental difference between the two studies will be addressed in the results section of this study.

It has been suggested that four patterns are used in language transfer as a language learning strategy when learning vocabulary; that L2 learners, especially beginning learners, use an equational framework (words in II equal words in L2) to associate new vocabulary words in the TL with preexisting NL vocabulary; and that as L2 learners progress, they become increasingly aware that the equational framework does not always hold true 
semantically. Accordingly, where language transfer occurs, and strategies such as avoidance are not being used, performance errors are expected to occur. Furthermore, beginning learners are expected to produce more lexico-semantic errors than advanced learners because it is predicted that beginning learners rely more on an equational framework of a one-to-one correspondence of words.

In order to address these notions the following hypotheses are proposed for this study:

Hypothesis 1: There is a hierarchy of difficulty to perform the four lexico-semantic patterns. On a scale from least difficult to most difficult, the patterns are congruence, convergence, divergence, and semantic gap.

Hypothesis 2: Beginning learners will demonstrate a higher frequency of lexico-semantic errors on the semantics test when the lexico-semantic patterns of convergence, divergence and semantic gap apply. This is due to the beginning learner's reliance on congruence, an equational framework where $\mathrm{L} 1=\mathrm{L} 2$, as a communication strategy.

Hypothesis 3: Advanced learners will demonstrate a lower frequency of lexico-semantic errors on the semantics test when the lexico-semantic patterns of convergence, divergence, and semantic gap apply. 
Hypothesis 4: The hierarchical order of the four lexico-semantic patterns will be the same in an acquisition poor environment as in an acquisition rich environment although measured performance (eg, number of errors) may differ.

The independent variable is the learner's proficiency level: a beginning group and an advanced group.

The dependent variable is the semantics test (Takahashi, 1984) designed to evaluate the correspondence between Japanese lexical items and English lexical items, based on congruence, convergence, divergence and semantic gap.

\section{Instrument}

The current study uses a lexico-semantics test developed by Takahashi (1984) to determine the hierarchical order of the four lexico-semantic patterns and their relative difficulty for beginning and advanced level students. It was developed to measure the use of lexico-semantic transfer patterns by native Japanese speakers studying EFL. The semantics test is composed of $a$ verb test and an adjective test. The verb test and the adjective test each consists of 28 fill-in-the-blank sentences for a total of 56 sentences. The verb test and the adjective test comprise Japanese sentences, each 
corresponding to a translated English sentence with one word omitted (see appendix A). The omitted word is a verb in the verb test and an adjective in the adjective test. The students' task was to fill in the blank of the English sentence with the word they felt best completed the Japanese to English translation.

\section{Validity}

There are two areas of validity to address: content validity and statistical validity (Garrett, 1962). content validity is said to be sound when the test deals directly with the area described by the inventory. The test in the current study deals directly with the measure of a learner's use of the lexico-semantic patterns. The statistical validity of tests "is determined by their correlations with various independent criteria" (Garrett, p.72, 1962). The present study applies several statistical tests to the lexico-semantics test to determine the statistical validity of the hypotheses.

Two points are considered at this time in order to address both the content and the statistical validity of the lexico-semantics test. First, Takahashi conducted two preliminary studies to establish a set of words that corresponded to the lexico-semantic patterns. The lexico-semantics test then incorporated the cue words into sentences to test the learner's use of the lexico- 
semantics patterns. It can be said that the test was created to deal directly with the area described in the inventory. Thus, the present study does possess content validity. Second, two statistical tests and a statistical measure are applied to determine the validity of the results: a Matrix of Spearman Correlation Coefficients Test, a T-test, and the comparison of means. The Matrix of Spearman Correlation Coefficients Test indicates whether the four patterns are distinct from one another; the T-test evaluates whether the difference between data is significant; and the comparison of mean averages suggests whether one pattern is used more often than the other. Thus, the current study does posses statistical validity. However, another aspect must be considered to establish the validity of the lexico-semantics test used in the present study: whether a test designed for an acquisition poor environment can be used to measure the same factors in an acquisition rich environment. Takahashi administered the lexico-semantics test in an acquisition poor environment, Japan. Also, the test was created to test students in an acquisition poor environment. However, there is no indication in Takahashi's study that the lexico-semantics test would be any different if she intended to administer the test in an acquisition rich environment. While it is true 
that an acquisition poor environment may not provide a learner with learning opportunities that may be present in an acquisition rich environment, the patterns and the level of the students are nonetheless the same in both environments. Takahashi's instrument possesses content and statistical validity and is a valid instrument for the present study.

In addition, since it is partially the intention of the present study to determine differences between an acquisition rich environment and an acquisition poor environment, then it seems reasonable to explore that relationship using the same instrument as Takahashi in an acquisition rich environment.

\section{Reliability}

Reliability involves the extent to which a measure is consistent and replicable. If the procedures of measure used within the semantic differential are explicit and can be replicated, the instrument possesses surface reliability (Osgood, Tannenbaum, Suci, 1975).

Takahashi addresses the reliability of her measure by suggesting that the combination of the two preliminary tests to establish the cue words, Tanaka's research on the four semantic patterns, Takahashi's careful development of supporting research on such categories as collocational restrictions and features, and the use of such categories to formulate the 
instrument, is sufficient to account for the reliability of the test.

Takahashi worked with a range of student proficiency levels, from beginning through advanced. My subject groups draw similarly from a beginning group and an advanced group. Even though a different tool was used in the current study to establish the participant's proficiency levels, there is every reason to believe that similar populations will respond similarly, thus reinforcing the test's reliability.

For my purpose, the test appears reliable with only one possible exception. Because Takahashi never administered the test in an acquisition rich environment, this may affect the test's overall reliability. However, in my opinion, this is not a strong enough reason not to use Takahashi's instrument. If anything, it seems more compelling to put this test to task in another environment.

The test possesses sufficient reliability as evidenced by Takahashi's discussion and the test's pilot testing to be used as the instrument of choice for the present study. Moreover, given that the instrument of the current study is valid and reliable, then it is sensible to assume correlations made between the beginning group and the advanced group will also be reliable. 
SUBJECTS

\section{General Description}

The subjects in this study are native speakers of Japanese, enrolled in a university or college ESL program at an advanced or intermediate level of their respective establishments. All the subjects took a TOEFL test or a Michigan test no more than six months prior to taking the lexico-semantics test. There is no discrimination for age or gender nor is there any consideration for how long a student has been living in the United States. All the subjects (a total of 55) volunteered for the test.

\section{Beginning Group and Advanced Group}

Takahashi's study consisted of three groups: beginning, intermediate, and advanced, with 100 Japanese students in each group. The current study consists of two groups, with 27 students in the beginning group and 28 students in the advanced group. It was determined that a sample size of about 30 participants was large enough to yield accurate results. The two groups used in the current study cover the extreme parameters of the Takahashi's study; it is believed that the results of the present study will reflect the range of students used in Takahashi's study. The following is a summary of the beginning and advanced groups. 
1. The beginning group consists of beginning and lowerintermediate ESL students with TOEFL scores of 430 or less or Michigan scores of 52 or less.

2. The advanced group consists of upper-intermediate and advanced ESL students with TOEFL scores of 441 or more or Michigan scores of 58 or more.

\section{Procedure}

Sixty-four native Japanese speakers, learning English and attending ESL programs, were tested using the lexico-semantics test. Two class levels were approached (advanced and intermediate) at six college ESL programs. The participants initially qualified according to their intermediate or advanced class levels in their respective schools. Each participant, however, was reclassified in the beginning group or the advanced group according to the student's Michigan or TOEFL test score. The scores were obtained from school records and, in some cases, directly from the students when they were able to remember their scores. Michigan scores were equated to TOEFL scores using a TOEFL conversion chart provided by Portland State University's ESL program (see Appendix B). TOEFL scores of 430 and below are considered a beginning and lower intermediate level. TOEFL scores of 441 and above are considered an upper intermediate and advanced level. A ten point buffer is provided between the two groups in order to more 
distinctly define the groups and minimize overlapping performance levels. Accordingly, nine of the sixty-four participants are not included in the study because of one or more of the following reasons: 1) the participant's TOEFL or Michigan score could not be obtained or confirmed; and/or 2) the participant's score fell within the ten point buffer. The final total number of participants in this study is fifty-five. Arrangements were made with the ESL directors of each of the six participating schools to introduce the purpose and format of the test to the instructors. A packet containing an introduction to the test was sent to each ESL director of the participating college or university. The packet contained an introduction to the thesis topic, a sample question and sample instructions of the semantic test, a release form, and a summary of what was expected from the participants. After each director had reviewed the material and presented the packet to his or her staff, arrangements were made to administer the test to the students. The test was administered by the researcher to all the participants of a school at the same time, either after regular classes at a prearranged place or during regular class time convenient for all parties involved. An exception to this was when the test was administered by ESL instructors to their respective classes while the 
researcher circulated throughout the classrooms to monitor the ESL instructors and participants. To maintain continuity, the instructors were provided instructions similar to those used by the researcher in previous testing sessions.

The general procedure for administering the test was as follows: first an explanation of the release form was presented to the students. Then the instructions were read out loud by the researcher or by the teacher as the students read along. The instructions provided a sample question identical to the format used in the test in order to familiarize the students with the format of the test before they began the semantics test. The participants were told the test was designed to measure vocabulary skills without any mention of the hypotheses for the study. All the students were made aware that their participation was strictly voluntary. Questions about the test were answered any time before, during or after the test. The participants were allowed 20 to 30 minutes to complete the test. In all cases, this proved to be ample time for the participants to complete the test.

\section{Data Analysis}

1) Students self-reported on the demographics section of the instrument, providing either a TOEFL or a Michigan test score. Based on these scores and confirmation of 
the scores, the subjects were separated into three groups: the beginning group, the advanced group and the buffer group.

2) The buffer group was eliminated from further study. The buffer group included participants with scores from 431 to 440 on the TOEFL test or from 52 to 57 on the Michigan test. The buffer group also included participants without recent or reliable TOEFL or Michigan test scores.

3) Individual item analyses for all 55 subjects were conducted for each of the 56 sentences.

4) In addition to item number, each sentence was labelled and grouped according to its lexico-semantic pattern: CG--congruence, CV--convergence, DV--divergence and SG--semantic gap, i.e., all semantic gap sentences were grouped together.

In sum, there is a master file for the beginning group and the advanced group. The master file represents the lexico-semantic patterns of each sentence, it divides the verb responses from the adjective responses, and it provides the type of responses and the total number of responses for each sentence. 


\section{CHAPTER IV}

\section{DATA ANALYSIS}

Chapter III described the methodology used in study. The present chapter outlines and discusses the quantitative results of the study.

Relative Difficulty of the Four Patterns

Verb Test Results. Figure 1 indicates, in graph form, the average performance (mean) for the beginning group and the advanced group according to the results of the verb test. 


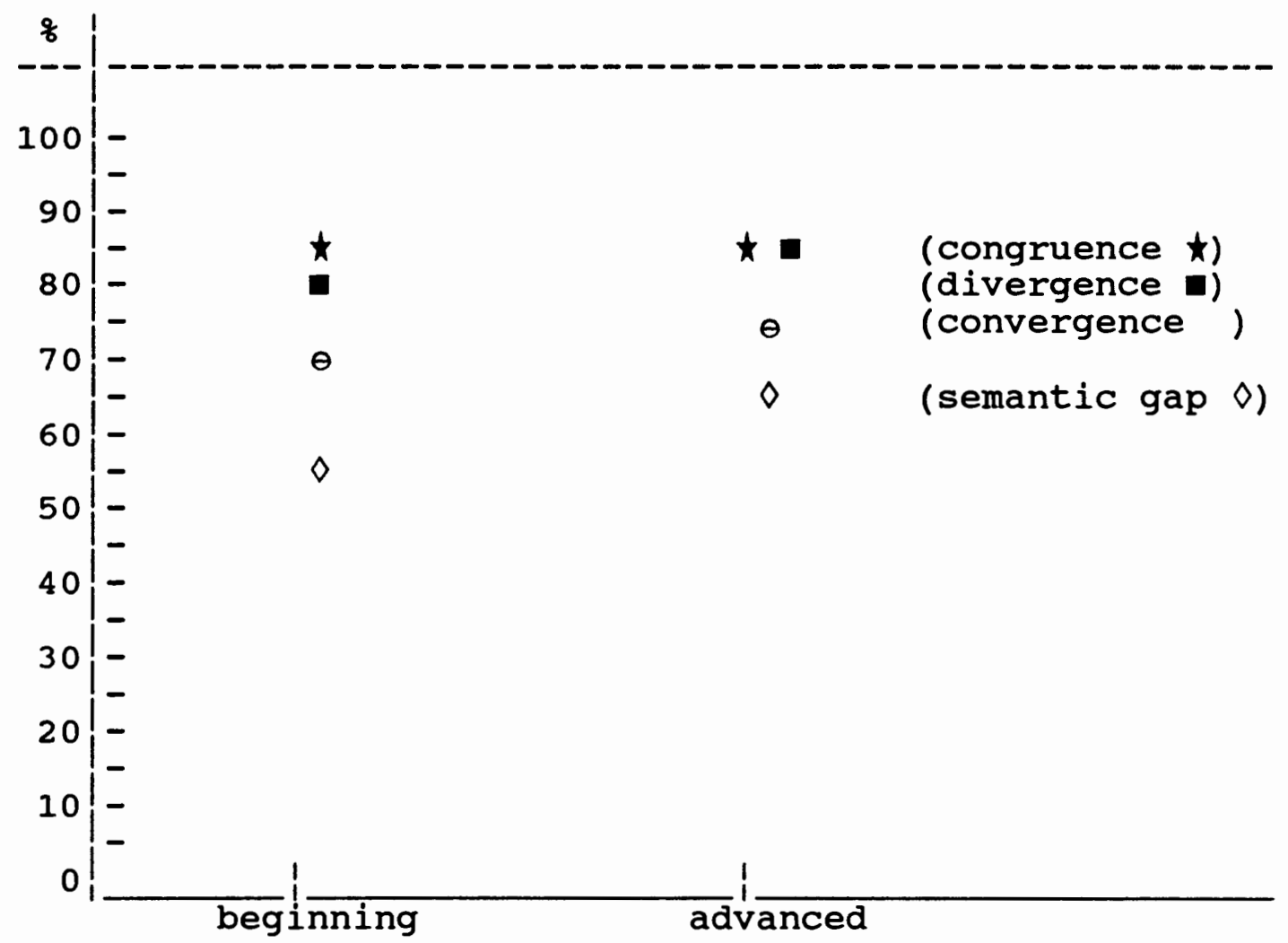

Figure 1. Average performance on the verb test for the beginning and the advanced group.

Summary of figure 1:

BEGINNING GROUP

Congruence: $\quad 86 \%$

Divergence: $\quad 80 \%$

Convergence: $70 \%$

Semantic gap: $53 \%$
ADVANCED GROUP

Congruence: $\quad 86 \%$

Divergence: $\quad 83 \%$

Convergence: $76 \%$

Semantic gap: $63 \%$

Figure 1 indicates that semantic gap is the most difficult pattern to perform irrespective of level of study. This is evident from the average score for semantic gap for the beginning group (53\%) and for the advanced group (63\%). A low mean average indicates a 
Figure 1 also suggests congruence is the least difficult pattern for the beginning group and the advanced group to perform. This is indicated by the average score of congruence (86\%), which is greater than any other score in the beginning and the advanced group. This supports Hypothesis 1, which proposed that congruence would be the least difficult pattern to perform.

The second least difficult pattern for the beginning ( $80 \%$ ) and advanced group ( $83 \%$ ) to perform is divergence. This contradicts part of hypothesis 1, in that convergence was predicted to be the second least difficult pattern to perform. However, as indicated, the results do not support this notion.

Convergence is the third most difficult pattern to perform for both groups. This again, contradicts the hierarchical order proposed in the present study. As mentioned above, hypothesis 1 predicted that divergence, not convergence, would be the third most difficult pattern to perform.

In sum, the verb test results for the beginning and the advanced group do not entirely correspond with the hierarchical order of the four lexico-semantic patterns predicted by hypothesis 1 . The relationship of the patterns will be discussed in greater detail later in this thesis. 
The rank order of the four patterns for the beginning group and the advanced group, according to the results in figure 1, for the verb test, are as follows:

1. Beginning group: $\mathrm{CG}>\mathrm{DV}>\mathrm{CV}>\mathrm{SG}$

2. Advanced group: $\mathrm{CG}>\mathrm{DV}>\mathrm{CV}>\mathrm{SG}$

Adjective Test Results. Figure 2 indicates the average performance (mean) for the adjective test for the beginning group and the advanced group in graph form.

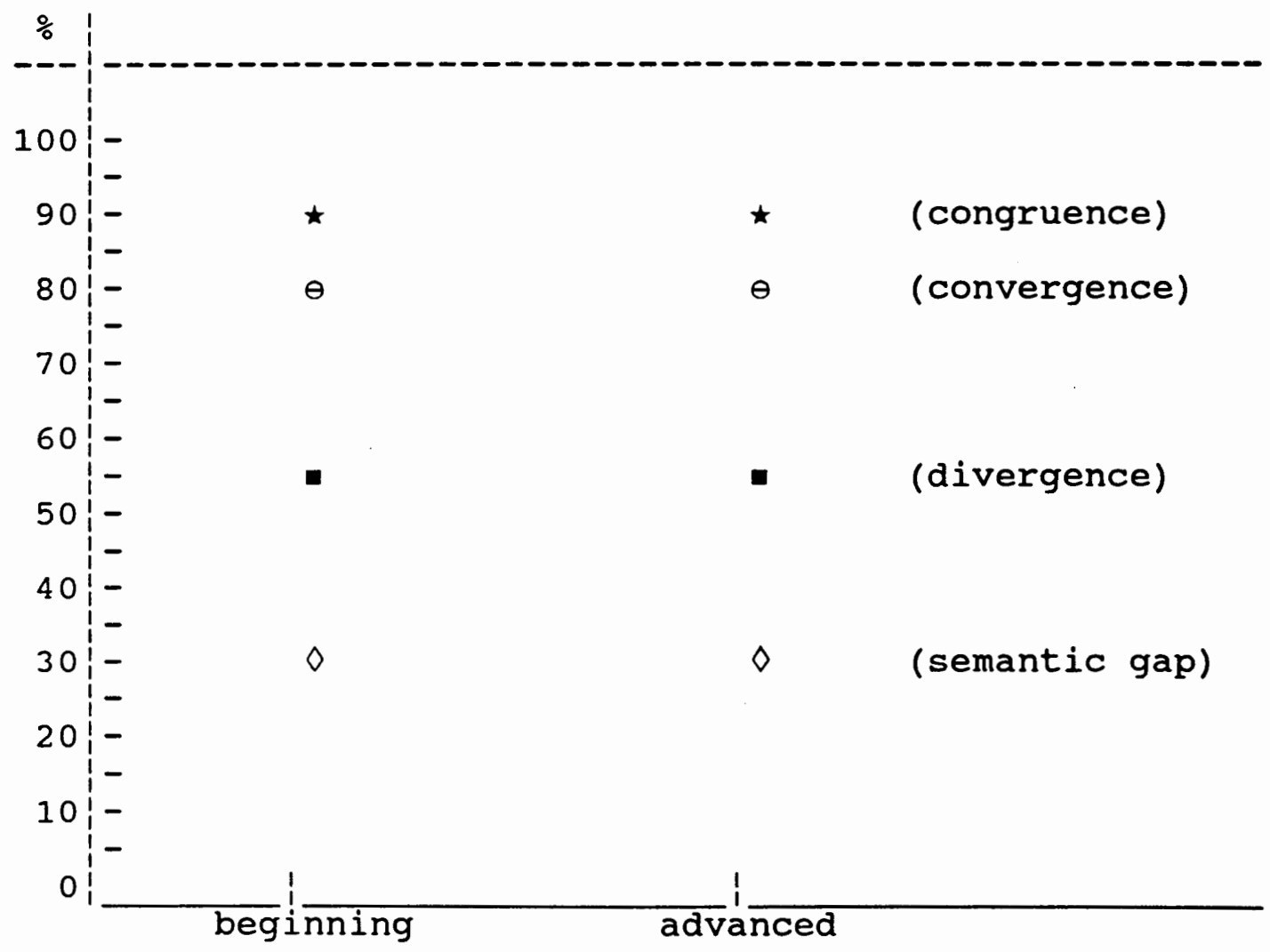

Figure 2. Average performance on the adjective test for the beginning and the advanced group. 
Summary of figure 2:

BEGINNING GROUP

ADVANCED GROUP

Congruence: $88 \%$

Congruence: $88 \%$

Convergence: $78 \%$

Convergence: $79 \%$

Divergence: $\quad 53 \%$

Divergence: $\quad 54 \%$

Semantic gap: $31 \%$ Semantic gap: $28 \%$

The results in figure 2 indicate that the

acquisition of the congruence pattern is the least

difficult for both groups: beginning group (88\%),

advanced group $(88 \%)$. Figure 2 also indicates that

semantic gap is the most difficult pattern for both

groups to perform: beginning group (31\%), advanced group

(28\%). In addition, figure 2 illustrates that, for the beginning group, convergence (78\%) is less difficult than divergence (53\%). The same order is indicated for the advanced group: convergence (79\%) is less difficult than divergence (54\%).

The beginning group and the advanced group each maintained the predicted hierarchical order proposed in hypothesis 1 with respect to adjectives. Later in this chapter, we will discuss whether or not the differences between the corresponding patterns in the beginning group are significantly different from those in the advanced group. In other words, we will determine if the advanced group outperformed the beginning group as predicted by hypotheses 2 and 3 of this study. To summarize, both groups followed the proposed 
hierarchical order in hypothesis 1, where congruence is the least difficult and semantic gap is the most difficult pattern to perform. The results are summarized as follows:

1. Beginning group: $\mathrm{CG}>\mathrm{CV}>\mathrm{DV}>\mathrm{SG}$
2. Advanced group: $\mathrm{CG}>\mathrm{CV}>\mathrm{DV}>\mathrm{SG}$ SIGNIFICANCE OF THE FOUR PATTERNS

The above section considered the mean of the four lexico-semantic patterns in order to indicate the actual hierarchical order of the patterns. This section first considers if the lexico-semantic patterns are four distinct patterns, and then determines if there is a significant difference between the corresponding patterns by comparing the two groups. This will indicate whether one group outperformed the other group.

\section{Four Distinct Patterns}

While Tanaka proposed that three patterns were appropriate for his study on prepositions, Takahashi indicated that four lexico-semantic patterns were required for her study on verbs and adjectives. As explained earlier, this is because Takahashi tested content words which, unlike prepositions, demonstrate the congruence pattern between the NL and the TL. Matrix of Spearman Correlation coefficients tests are used in the current study to determine if the patterns 
are four distinct patterns (tables I and II below). The results indicate that the correlation coefficients are low (not near either -1 or +1 ). This is interpreted to mean that the four patterns are different from one another and should be considered four distinct patterns. The reasoning is this: if two so-called distinct patterns were actually two names for one real pattern, then the student who used one pattern often would use the other pattern often as well. This would be reflected in a high correlation coefficient (near +1 ). On the other hand, if two patterns really are different, then a student who used one pattern would not necessarily use the other pattern. This would be reflected in a correlation coefficient closer to 0 (not near +1$)$. This is what is seen below in tables $I$ and II.

\section{TABLE I}

MATRIX OF SPEARMAN CORRELATION COEFFICIENTS BEGINNING AND ADVANCED ADJECTIVE TEST

$\begin{array}{lll}A C G & A C V & A D V\end{array}$

$A C G$

$A C V$

ADV

1.000

ASG

0.308
-0.133
0.145

1. 000

0.063

0.137

1.000

0.279

1.000 
TABLE II

MATRIX OF SPEARMAN CORRELATION COEFFICIENTS BEGINNING AND ADVANCED VERB TEST

$\begin{array}{llll}\text { VCG VCV VDV VS } & \text { VC }\end{array}$

VCG $\quad 1.000$

VCV $\quad-0.122$

VDV -0.123

VSG

0.115

1.000

0.202

0.292

1.000

0.273

1.000

\section{Significant Difference between the Two Groups}

This section compares the corresponding lexicosemantic patterns of the two groups to determine if there is any significant difference between the beginning group and the advanced group. This will indicate if one group outperformed the other group. Hypotheses 2 and 3 suggested that the advanced group would outperform the beginning group in the adjective test and the verb test.

Adjective Congruence. Table III compares the adjective test results of the beginning group and the advanced group. The median indicates that both groups demonstrated similar success using the congruence pattern; adjective congruence for the beginning group and the advanced group was successfully used approximately $86 \%$. The mean for the beginning group and the advanced group (approximately 88\%) also indicates that both groups performed similarly. In addition, the T-test results show that $p>0.05$, which indicates there is no significant difference between the performance of 
the beginning group and the advanced group.

TABLE III

ADJECTIVE CONGRUENCE; A COMPARISON BETWEEN THE BEGINNING GROUP AND THE ADVANCED GROUP

GROUP

Mean

Median
BEGINNING

0.883

0.857
ADVANCED

0.877

0.857

T-test: $\mathrm{p}=.8213>0.05$

In sum, the results suggested by median, mean, and the T-test all indicate there is no significant difference between the two groups.

Adjective Convergence. Table IV compares the adjective convergence test results of the beginning and the advanced group. The median indicates that both groups demonstrated similar success using the convergence pattern; adjective convergence for the beginning group and the advanced group was successfully used 0.857 of the time or approximately $86 \%$. The mean for the beginning group and the advanced group is also similar for both groups, approximately $78 \%$ and $79 \%$, respectively. In addition, the $\mathrm{T}$-test results show that $p>0.05$, which indicates there is no significant difference between the performance of the beginning group and the advanced group. 
TABIE IV

ADJECTIVE CONVERGENCE; A COMPARISON BETWEEN THE BEGINNING GROUP AND THE ADVANCED GROUP

GROUP

Mean

Median
BEGINNING

$$
0.783
$$

0.857
ADVANCED

0.791

0.857

T-test: $\mathrm{p}=.4288>0.05$

In conclusion, both groups performed similarly in respect to convergence in the adjective test irrespective of group level. This indicates that both groups performed similarly.

\section{Adjective Divergence. Table V compares the} successful use of divergence by the two groups in the adjective test. The median indicates that both groups had relatively the same success using the divergence pattern; adjective divergence for each group was successfully used 0.571 of the time or approximately $57 \%$. The mean for the beginning group and the advanced group, $53 \%$ and $54 \%$ respectively, suggests the groups performed similarly. Furthermore, the results of the T-test show that $p>0.05$, which indicates there is no significant difference between the performance of the two groups. 
TABLE V

ADJECTIVE DIVERGENCE; A COMPARISON BETWEEN THE BEGINNING GROUP AND THE ADVANCED GROUP

GROUP BEGINNING ADVANCED

$\begin{array}{lll}\text { Mean } & 0.534 & 0.540 \\ \text { Median } & 0.571 & 0.571\end{array}$

T-test: $\mathrm{p}=.8531>0.05$

In conclusion, the results of the $\mathrm{T}$-test indicate there is no significant difference between the two groups with respect to divergence in the adjective test. This is supported by the median and the mean. This supports the notion that the beginning and the advanced group performed divergence equally well in the adjective test irrespective of group level.

Adjective semantic Gap. Table VI illustrates the successful use of semantic gap in the beginning and the advanced group concerning the adjective test. The median indicates that both groups had similar success using the semantic gap pattern; adjective semantic gap for the beginning group and the advanced group was successfully used approximately $29 \%$ of the time. Furthermore, the mean for the beginning group is approximately $31 \%$, and approximately $28 \%$ for the advanced group, which suggests the two groups performed similarly. T-test results conclude $\mathrm{p}>0.05$, which indicates the beginning group and the advanced group 
performed equally well with no significant difference between the two groups.

TABLE VI

ADJECTIVE SEMANTIC GAP; A COMPARISON BETWEEN THE BEGINNING AND THE ADVANCED GROUP

GROUP BEGINNING ADVANCED

Mean

0.306

0.275

Median

0.285

0.285

T-test: $\mathrm{p}=7917>0.05$

In sum, the results indicate there is no significant difference in the performance of the two groups with respect to semantic gap for the adjective test. This is supported by the median, mean, and the T-test.

Verb congruence. Table VII illustrates the successful use of congruence in the beginning and the advanced group for the verb test. The median indicates that both groups had similar success using congruence; verb congruence for the beginning group $(0.857)$ and the advanced group $(0.851)$ was successfully used approximately $86 \%$ and $85 \%$, respectively. The mean for the beginning group and the advanced group are approximately $86 \%$. In addition, the $\mathrm{T}$-test results show that $p>0.05$. This indicates there is no significant difference. Therefore, it can be said that the 
beginning and the advanced group performed equally well.

TABLE VII

VERB CONGRUENCE; A COMPARISON BETWEEN

THE BEGINNING AND THE ADVANCED GROUP

GROUP

Mean

Median
BEGINNING

0.863

0.857
ADVANCED

0.862

0.851

T-test: $p=7169>0.05$

In conclusion, the median, mean, and the results of the $T$-test indicate there is no significant difference between the performance of the two groups.

\section{Verb Convergence. Table VIII illustrates the} successful use of convergence in the two groups concerning the verb test. The median indicates that both groups had the same success using the congruence pattern; verb convergence for the beginning and the advanced group was successfully used 0.714 of the time or approximately $71 \%$. The mean for the beginning group is approximately $70 \%$, and approximately $76 \%$ for the advanced group. This also shows a similar use of the verb convergence pattern. A T-test concluded that $\mathrm{p}>$ 0.05 , which indicates that the beginning and the advanced group performed equally well. 
TABLE VIII

VERB CONGRUENCE; A COMPARISON BETWEEN

THE BEGINNING GROUP AND THE ADVANCED GROUP

GROUP

Mean

Median
BEGINNING

0.703

0.714
ADVANCED

0.755

0.714

T-test: $p=.1801>0.05$

In sum, the T-test indicates there is no significant difference in the performance of the two groups in the verb test for the congruence pattern. This is further supported by the results of the median and the mean.

Verb Divergence. Table IX shows how divergence is used in the two groups concerning the verb test. The median indicates that the beginning and the advanced group used the divergence pattern 0.857 or $86 \%$ of the time. The mean for the beginning group is approximately $80 \%$ and approximately $83 \%$ for the advanced group. This suggests that the beginning and the advanced group performed equally well. Furthermore, T-test results show that $p>0.05$, which indicates there is no significant difference between the performance of the two groups. 
TABLE IX

VERB DIVERGENCE; A COMPARISON BETWEEN THE BEGINNING GROUP AND THE ADVANCED GROUP

GROUP

Mean

Median
BEGINNING

$$
0.799
$$$$
0.857
$$

ADVANCED

0.831

0.857

T-test: $\mathrm{p}=.1614>0.05$

In conclusion, the results indicated by the T-test and supported by the median and the mean suggest there is no significant difference between the performance of the beginning and the advanced group with respect to divergence for the verb test.

Verb semantic Gap. Table $X$ how semantic gap is used in the beginning and the advanced groups in the verb test. The median indicates that both groups had relatively the same success using the semantic gap pattern; verb semantic gap for each group was successfully used 0.571 of the time or approximately 57\%. The mean for the beginning group is approximately 53\%, and for the advanced group it is approximately $61 \%$. In addition, the results of the $\mathrm{T}$-test show that $\mathrm{p}>$ 0.05 which suggests there is no significant difference between the two groups. However. the results are .0781, which is close to 0.05 . This indicates that there is a non-significant "tendency" for the advanced students to use semantic gap more than did the beginning students. 
TABLE $X$

VERB SEMANTIC GAP; A COMPARISON BETWEEN

THE BEGINNING GROUP AND THE ADVANCED GROUP

GROUP

Mean

Median
BEGINNING

$$
0.529
$$

0.571
ADVANCED

0.607

0.571

T-test: $p=.0781>0.05$

In sum, the results indicated by the T-test and supported by the median, and the mean, suggest the beginning and the advanced group use verb semantic gap equally well with a tendency for the advanced group to use semantic gap more often. Since it is a nonsignificant tendency, we can conclude that the two groups performed equally well.

\section{Summary}

The results strongly indicate that the beginning and the advanced group used the four patterns equaliy well without any significant difference. This is contrary to hypotheses 2 and 3 , which proposed that the advanced group would outperform the beginning group. This point will be further explored in Chapter $V$. 


\section{CHAPTER V \\ CONCLUSIONS, LIMITATIONS, SUGGESTIONS, AND TEACHING APPLICATIONS}

This chapter examines the hypotheses in the current study with respect to the hierarchical order of difficulty of the four lexico-semantic patterns, the comparative performance of beginning and advanced students, and the maintenance of the hierarchical order proposed in Takahashi's study in an acquisition rich environment. In addition, limitations, suggestions and recommendations for teaching applications are given.

\section{Conclusions and Limitations}

Hypothesis 1: There is a hierarchy of difficulty to perform the four lexico-semantic patterns. On a scale from least difficult to most difficult, the patterns are congruence, convergence, divergence, and semantic gap.

The results of the current study indicate that semantic gap is the most difficult pattern to use because, in comparison to the other three lexicosemantic patterns, semantic gap was the least successfully completed pattern in both the verb and the adjective tests for the beginning and the advanced 
groups. This conclusion is partly drawn on the notion that the pattern most easily produced is the pattern most frequently used successfully, and the pattern least easily produced is the pattern less frequently used successfully.

The results in the present study support the notion that semantic gap requires the learner to completely restructure the meaning of a word by learning the appropriate collocational restrictions for the new word through a trial and error basis. Accordingly, semantic gap maintains the order of difficulty predicted by hypothesis 1 .

In addition, hypothesis 1 proposes that congruence is the least difficult pattern to use. The results of the current study indicate that, in comparison to the other lexico-semantic patterns, congruence is the most successfully completed pattern in both the verb and the adjective tests for both groups. It can be said, therefore, that congruence conforms to the order of difficulty proposed by hypothesis 1 .

Hypothesis 1 also suggests that convergence is less difficult to use than divergence. This is suggested by the notion that the process of blending loverlooking distinctions), required in the use of convergence, is accomplished more easily than sifting (making new distinctions), which is required in the use of 
divergence.

The results indicate that convergence is easier to use than divergence for both groups in the adjective test. However, the verb test results indicate divergence is easier to perform than convergence, contrary to the original proposal. In other words, while the two groups follow the predicted order of difficulty in the adjective test, they fail to do so in the verb test.

It is interesting, however, that the results of the verb test results are the same as those found in Takahashi's study. Takahashi concludes that is because the process of blending (convergence) may be more difficult than originally predicted. She speculates that Japanese students are so accustomed to using Japanese convergence items that it makes it hard for them to think that two Japanese items correspond to one English item and, therefore, have difficulty adjusting to TL collocational restrictions. If so, then why does this only pertain to verbs? There is no clear answer to this question in the current study, but Takahashi suggests, and the results of the present study indicate, that only some restrictions placed on sentences are difficult for students to adjust to. Therefore, since the current study and Takahashi's study used the same instrument, it can be said that the students 
consistently demonstrate that blending (convergence), is not always easier than sifting (divergence).

Furthermore, the current study indicates that this holds true in an acquisition rich environment as well as an acquisition poor environment.

In conclusion, the results of the current study support the hypothesis that congruence is the least difficult pattern to use and semantic gap is the most difficult pattern to use. However, with respect to divergence and convergence, the hierarchical order is different for verbs than with adjectives. The present study supports Takahashi's results that verbs follow the hierarchical order of congruence, convergence, divergence, and semantic gap while adjectives follow the hierarchical order of congruence, divergence, convergence, and semantic gap.

Moreover, the hierarchical order of congruence, divergence (split), and semantic gap proposed by Tanaka is maintained throughout all three lexico-semantic studies regarding prepositions, verbs and adjectives. This implies that the four lexico-semantic patterns may be consistent across Iinguistic categories. However, since this has only been tested with Japanese and English subjects, more studies on other Iinguistic categories and languages are needed to conclude that these patterns and hierarchical order are limited 
universals or universals.

Hypothesis 2: Beginning learners will demonstrate a higher frequency of lexico-semantic errors on the semantics test when the lexico-semantic patterns convergence, divergence and semantic gap apply. This is due to the beginning learner's reliance on congruence, an equational framework ( $L 1=L 2)$, as a communication strategy.

According to the results, the beginning group performed as well as the advanced group in the adjective test and the verb test; in other words, the beginning group did not rely on the equational framework, congruence, any more than did the advanced group.

Therefore, hypothesis 2 is not supported by the results of this study. This notion will be discussed in more detail when discussing hypothesis 3 .

Hypothesis 3: Advanced learners will demonstrate a lower frequency of lexico-semantic errors on the semantics test when the lexico-semantic patterns of convergence, divergence, and semantic gap apply.

As mentioned above, the results of the present study suggest there is no significant difference between the performance of the beginning and the advanced group in the verb or the adjective tests. Performance is 
measured by the success rate with which learners use the lexico-semantic patterns.

It is difficult to understand why the beginning group performed as well as the advanced group in the present study, when the beginning group did not perform as well as the advanced group in the Takahashi study (1984). One explanation could be that the beginning group in the current study was a higher level group than the beginning group in the Takahashi study. However, the beginning and the advanced group of the present study were established according to one of two standardized tests, the TOEFL or the Michigan test. In addition, to assure that the advanced group was distinctly more advanced than the beginning group, a buffer was used to further separate the level of the subjects. It is likely, therefore, that the two levels were a beginning and an advanced group. Thus, it seemed reasonable to assume that the advanced group would outperform the beginning group, but, as indicated by the results, this did not occur.

It is also possible, however, that the instrument used in the present study did not test the population's full ability. Perhaps Takahashi designed the constructions and types of sentences for lower level advanced subjects. This suggests that the beginning learners would have been tested to their full range of 
ability, but the advanced students would have been tested to a limited extent because the sentences would have been too easy for the advanced subjects. To account for this, an expanded version of the lexicosemantics test, which includes more challenging sentence constructions and types for the advanced group, needs to be designed. While this would perhaps address this particular problem, it is not within the confines of the present study to accomplish this task.

Another explanation for the unexpected results is that the subjects in the present study had been Iiving in an acquisition rich environment. Therefore, the beginning group in the current study may have had more opportunity to restructure their hypotheses and thereby progress faster along a learning continuum than the students in the Takahashi study. Accordingly, it is possible that the beginning learners in this study were able to use the lexico-semantic patterns as well as the advanced learners and that this was reflected in the results of this study. This notion parallels the previous idea that the learners were not tested to their full potential. Therefore, expanding the lexicosemantics test would also address this latter hypothesis.

Another possibility for both groups performing similarly is that there were not enough subjects to 
yield accurate results. Takahashi's study included 300 participants. As mentioned in the Methodology Chapter of this study, it was determined that approximately thirty participants were necessary for accurate results. This goal was met with twenty-seven participants in the beginning group and twenty-eight participants in the advanced group. Therefore, while it is possible that the relatively low number of subjects could have interfered with the results, it seems that there should have been enough subjects to provide some accurate indication of the subjects' abilities.

To summarize the results, the two groups did not follow the predicted hierarchical order of the lexicosemantic patterns in the verb test. on the other hand, the two groups maintained the predicted hierarchical order in the adjective test. Convergence and divergence stayed between congruence and semantic gap throughout the study. This supports Tanaka's hierarchical order of congruence, divergence, and semantic gap.

Furthermore, although it was predicted that beginning learners would demonstrate more errors when using the patterns convergence, divergence and semantic gap, the results in this study indicate there is no significant difference between the beginning group and the advanced group. This may be due to the instrument's limited ability to fully test the advanced subjects. 
A number of possibilities have been introduced to account for these results, but it seems that a follow up study is required to fully determine the role of the lexico-semantic patterns in I2 vocabulary acquisition.

Nonetheless, based on what the current study has revealed, it can be concluded that the beginning group and the advanced group performed equally well and that two major factors could have influenced these results: the influence of an acquisition rich environment and the instrument's limited ability to measure the full range of the advanced learners in that environment.

Hypothesis 4: The hierarchical order of the four lexico-semantic patterns will be the same in an acquisition poor environment as in an acquisition rich environment.

The results of the present study indicate that the four lexico-semantic patterns maintain the same hierarchical order in an acquisition rich environment as in an acquisition poor environment. The order maintained in the present study is not the order originally predicted by Takahashi, but it is the same order concluded in Takahashi's study. The present study concludes that the hierarchical order for verbs is the same order found in Takahashi's study. Also, the current study concludes that adjectives follow the same 
hierarchical order predicted in the Takahashi study.

Accordingly, the present study concludes that although an acquisition rich environment does not affect the hierarchical order of the lexico-semantic patterns, the performance level of beginning learners may be drastically affected. In other words, an acquisition rich environment does seem to enhance the use of the lexico-semantic patterns for beginning learners.

\section{Suggestions}

The results of the present study could not determine if the advanced learners' abilities were fully tested in an acquisition rich environment because of possible limitations of the instrument. A follow up study using an expanded instrument could determine whether beginning learners and advanced learners progress proportionally along a language learning continuum with respect to the use of the lexico-semantic patterns.

Furthermore, a follow up study could firmly establish whether the lexico-semantic patterns are consistently used across linguistic categories.

\section{Teaching Applications}

The results of this study suggest that the four lexico-semantic patterns can serve as indicators of a learner's difficulties in I2 vocabulary acquisition, but 
they may not serve as reliable indicators of student levels or student progression in a language learning continuum. The patterns can indicate a student's specific problem if the teacher is familiar with the student's native language. If research in lexicosemantics were more extensive, then perhaps some generalities about lexico-semantics with respect to languages could be made. A teacher could then predict and account for general transfer problems students may have, depending on their native language, much like the way teachers use general knowledge about phonological systems. However, since this would require a wide body of research, which does not exist at this time, the use of lexico-semantics in the classroom is limited for now. At this time, it may be sufficient for a teacher to be aware that the interaction or transfer of semantic values between the native language and the target language can cause difficulties in learning and applying vocabulary. A teacher could note recurring difficulties and associate those with one of the four lexico-semantic patterns and consequently bring it to a learner's awareness. It is also possible that associating specific problems with lexico-semantic patterns may better enable a learner to self-monitor and perhaps be more selective when consciously assigning semantic values to a word. However, this seems highly 
impractical for acquiring and using a large body of vocabulary.

In conclusion, more research is needed concerning the practical use of the patterns in language teaching and language learning. Such an undertaking was not within the scope of this study, but I do hope the full value of lexico-semantic patterns can be realized in future research. 


\section{REFERENCES}

Brown, H. D. (1980). Principles of language learning and teaching. New Jersey: Prentice Hall.

Catford, J. (1968). Contrastive analysis and language teaching. In J. Alatis (Ed.), Contrastive Iinguistics and its pedagogical implications. Washington, D.C.: Georgetown University Press, 159-174.

Corrale, O., \& Cal, E. (1989). At a loss for words: The use of communication strategies to convey lexical meaning. Foreign Language Annals, 22 (3), 227-250.

Garrett, E. Henry. (1962). Elementary statistics. New York: David McKay Company, Inc.

Gass, S. (1988) . Second language vocabulary acquisition. Annual Review of Applied Iinguistics, ․, $92-106$.

Jakobovits, Leon, A. (1969). Second language learning and transfer theory: $A$ theoretical assessment. Language Learning, 19 (1), 55-86.

Krashen, S. (1989). We acquire vocabulary and spelling by reading: additional evidence for the input hypothesis. Modern Language Journal, 73, 440-464.

Lado, Robert. (1957). Linguistics across culture: Applied linguistics for language teachers. Ann Arbor: University of Michigan Press.

Laufer, B. (1990). Ease and difficulty in vocabulary learning: Some teaching implications. Foreign Language Annals, 23 (2), 147-155.

Lee, W. R. (1968). Thoughts on contrastive linguistics in the context of language teaching. In $J$. Alatis (Ed.), Contrastive Iinguistics and its pedagogical implications. Washington, D.C.: Georgetown University Press, 185-194. 
Osgood, C. H., Tannenbaum, P.H., \& Suci, G. J. (1975). The measure of meaning. Ann Arbor: University of Michigan Press.

Schachter, J. (1974). An error in error analysis. Language Learning, 24 (2), 205-214.

stockwell R. (1968). Contrastive analysis and the lapse of time. In J.Alatis (Ed.), Contrastive

linguistics and its pedagogical implications. Washington, D.C.: Georgetown University Press. 11-26.

Sugano, M. (1981). The idioms in Spanish language teaching. Modern Lanquage Journal, 65, 59-66.

Takahashi, T. (1984). A study on semantic transfer. Doctoral dissertation. University Microfilms Intermational: No. 8424272

Tanaka, S. (1983). Language transfer as a constraint on lexico-semantic development in adults learning a second lanquage in acquisition-poor environments. University Microfilms International: No. 8403288

Tanaka, S., \& Abe, H. (1984). Conditions on interlingual semantic transfer. On TESOL'84.

Tarone, E. (1980). Communication strategies, foreigner talk, and repair in interlanguage. Lanquage Learning, 30 (2), 417-431.

Thorndike, E. I. (1932), 1975 (revised edition). A teacher's word book of the twenty thousand words found most frequently and widely in general reading for children and young people. New York: Teachers College Press, Columbia University. 


\section{APPENDIX A}

\section{VERBS}

(1) What's that noise at the door? Did you it, too? [V, DV]

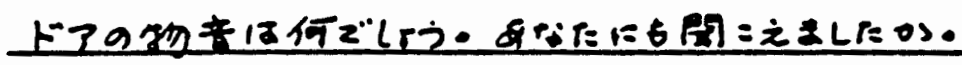

(2) I'II $[V, D V]$ to Professor Brown about this matter.

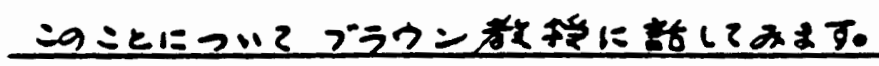

(3) My three-year-old son likes to pictures with colored pencils. [V, SG]

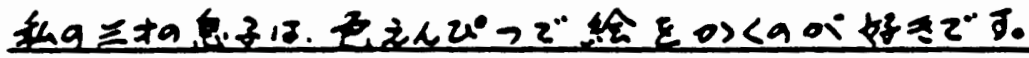

(4) Can you believe that Tom can up a whole bottle of wine? [V, CG]

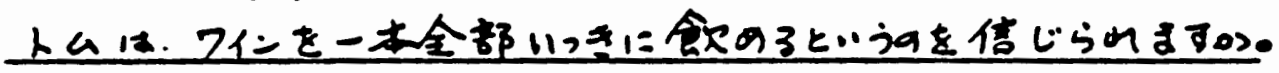

(5) If you want to dance well, please carefully how I dance. [V, DV]

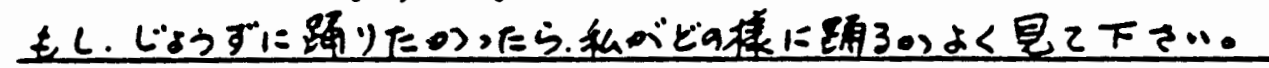

(6) Many young people coday children, but don't want to take care of them. [V,SG]

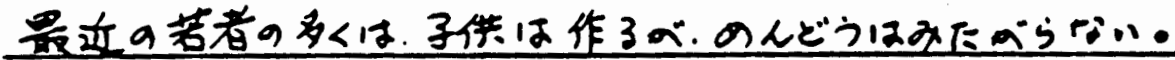

(7) When I get to Japan, I will $[\mathrm{V}, \mathrm{CG}]$

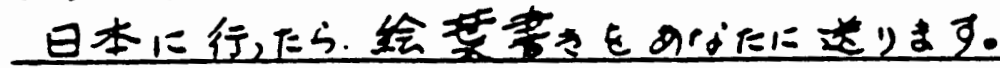

(8) I've never been to Ueno zoo. Would you me there next Sunday? [V, CV]

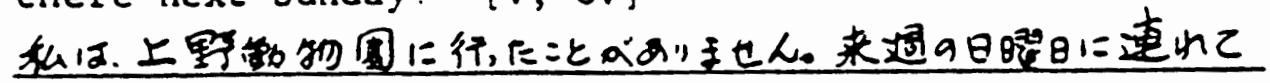
行,ていただけはせん○。 
(9) The farmers in this village are vegetabies.

[V, SG]

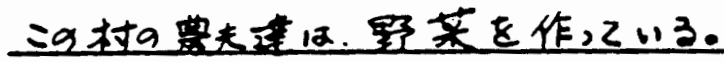

(10) I'm not asking you if you can a motorcycle, but if you have one. [V,SG] khid. arta゙

(11) We have to pay $\$ 280$ a month to this apartment. $[V, D V]$

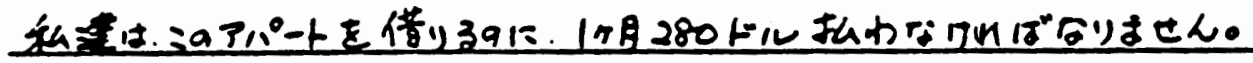

(12) Would you more slowly? I don't understand English very well. [V, DV]

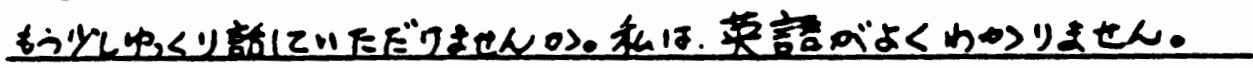

(13) I know you like meat, but you'd better more vegetables. [V, CG]

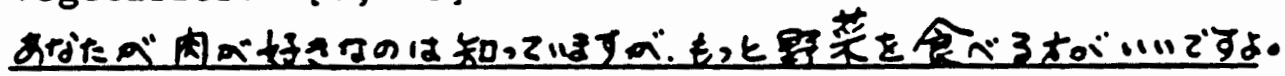

(14) Please some eggs while I make some coffee. $[\mathrm{V}, \mathrm{CV}]$

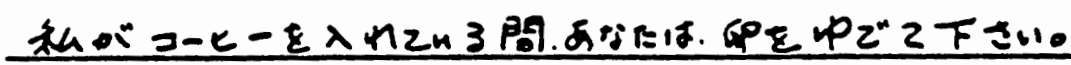

(15) I'm very thirsty. I want to a glass of water. [V, CG]

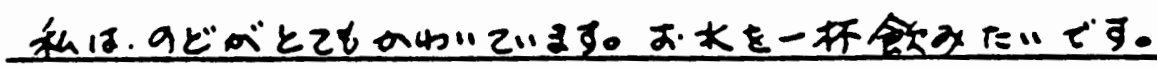

(16) I a strange dream last night. You were a princess in my dream. [V, SG]

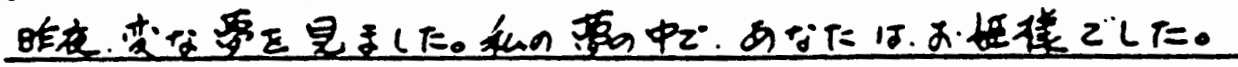


(17) Do you the music nextdoor? I think they are having a party. [V, DV ]

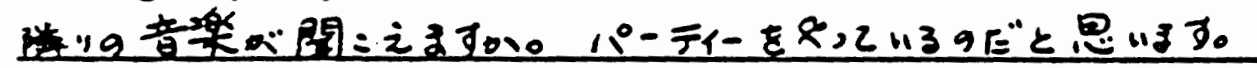

(18) Do you know that man who always that crazy hat? [V, CV ]

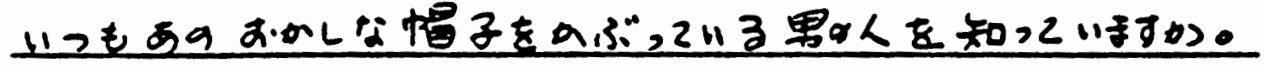

(19) Mary her husband some potatoes. [V, CG]

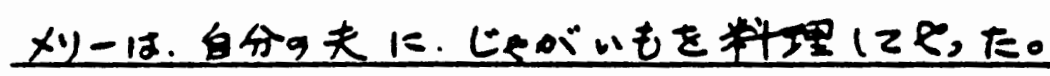

(20) Why don't you

if you have a headache? [V,SG]

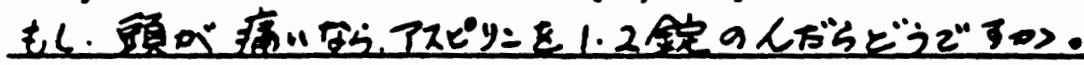

(21) You shouldn't the vegetables for too long. [V, CV]

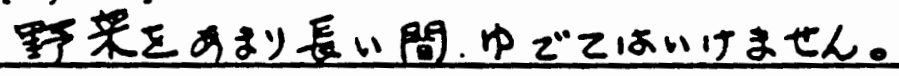

(22) I have to a telegram to my friend who is getting married soon. [V, SG]

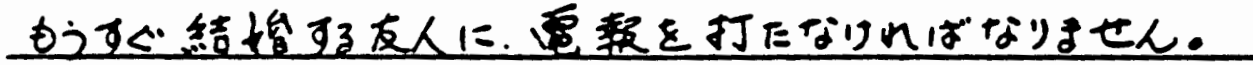

(23) Do you think this plant is good to $?[\mathrm{~V}, \mathrm{CG}]$

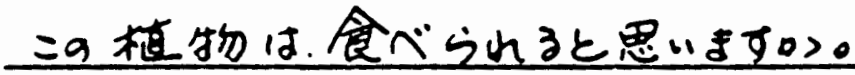

(24) When you visit us next time, please your chileren with you. $[\mathrm{V}, \mathrm{CV}]$

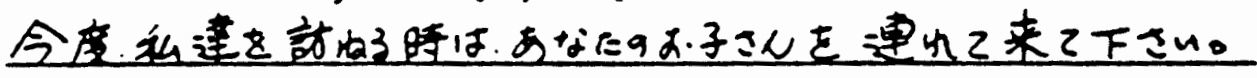


(25) Do you. the policeman over there? Ask him for directions. (V, DV !

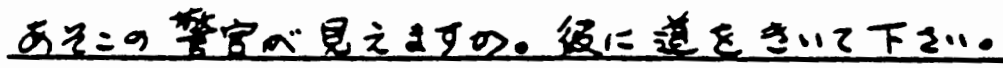

(26) You may think it's difficult, but for me it's easy to fish. [V, CG]

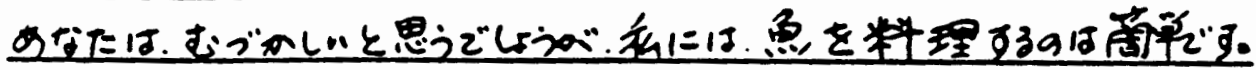

(27) Please taping it. $[\mathrm{V}, \mathrm{CV}]$

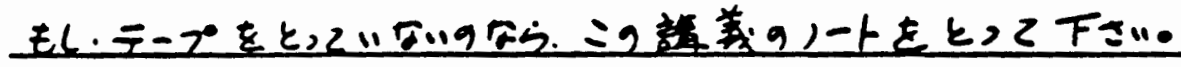

(28) Please these dishes away and wash them. $[\mathrm{V}, \mathrm{CV}]$

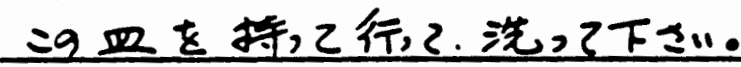

\section{ADJECTIVES}

(1) I have to go to the barber shop. My hair is getting too - [A, CG]

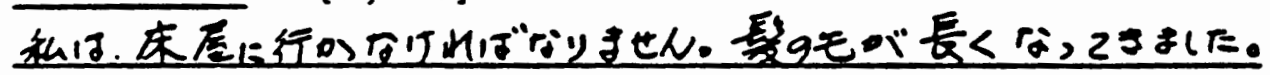

(2) This tree is so that we can't cut it down. [A, SG]

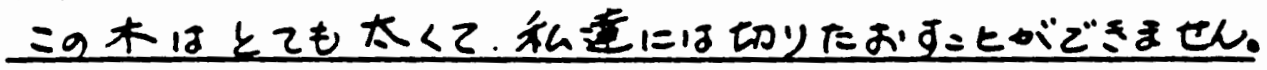

(3) You 'd better not buy those bananas. They are still - $[A, S G]$

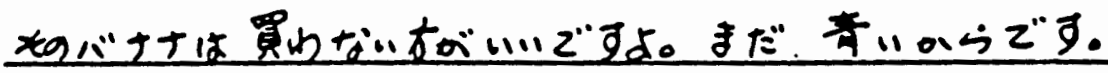


(4) Take this local train. It'il take a while, but you' 11 get there by noon. [A, DV]

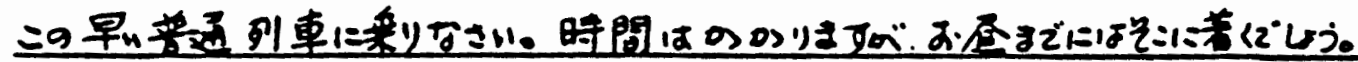

(5) My room is very , but it is the most comfortable place for me. [A, SG]

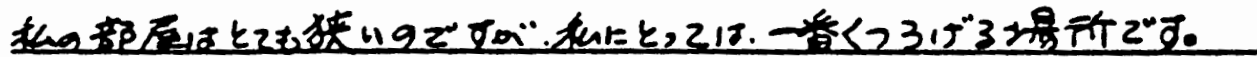

(6) The Browns and the Millers are our relatives. [A, DV]

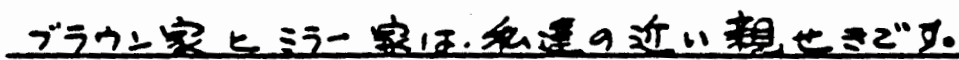

(7) The snow lay 3 feet on the street. [A, SG]

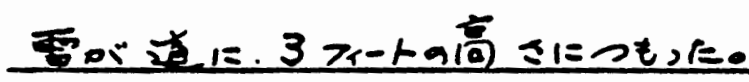

(8) I don't like this soup because it's too $[\mathrm{A}, \mathrm{CV}]$

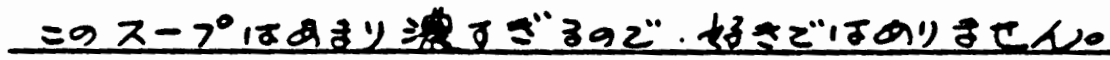

(9) Nobody can buy that car because the price is too - [A, DV ]

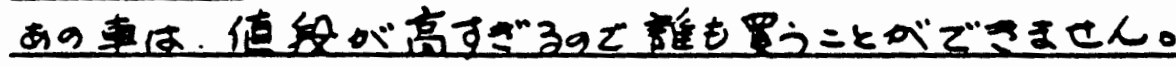

(10) Mary and Susan are friends of mine. $[A, C V]$

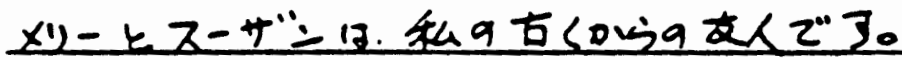

(11) This dress is too for me. Let me try the other one. [A, CG]

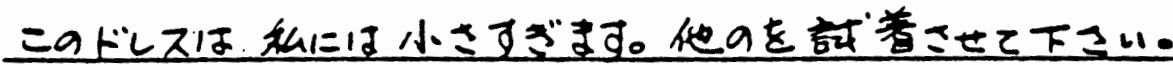


(12) This fishing rod is only 3 feet - $[A, C G]$

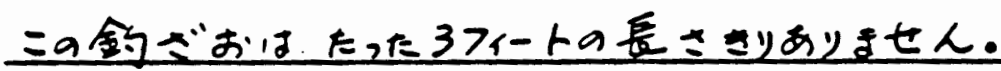

(13) His voice is so that it hurts my ears. [A, DV]

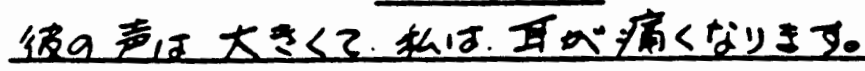

(14) My hands are getting - Let me warm them at the fire. [A, CV]

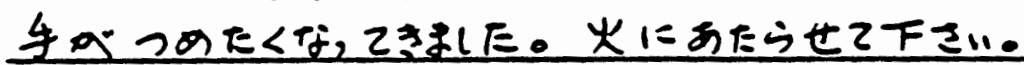

(15) This pond is very and it has many kinds of fish. $[A, C G]$

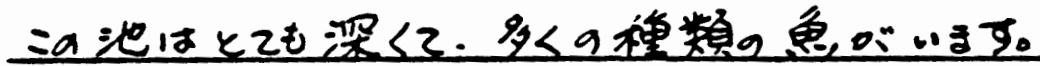

(16) He is tions. [A, DV ]

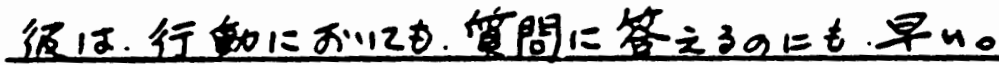

(17) My sister doesn't think she is pretty because her nose is very - [A, SG]

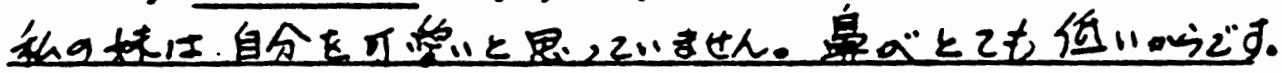

(18) Since I last saw my grandparents, they've gotten very - [A, CV]

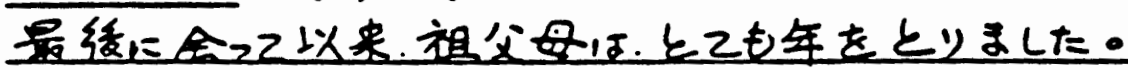

(19) I don't like this coffee. It's too [A, SG]

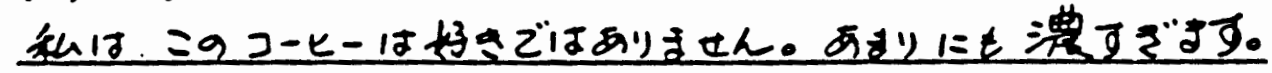

(20) Mary told me that the sky was always in California. [A, CG]

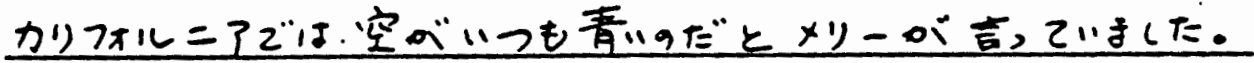


(21) You shouldn't play outside today. The wind is very - $[A \quad C G]$

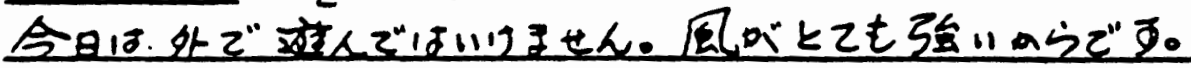

(22) I think we are going to have a very winter this year. [A CV]

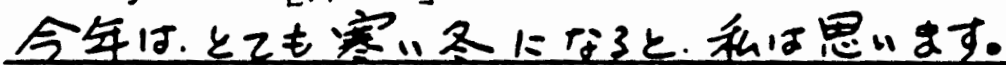

(23) I like the singer Barry White because his voice is very - [A, SG]

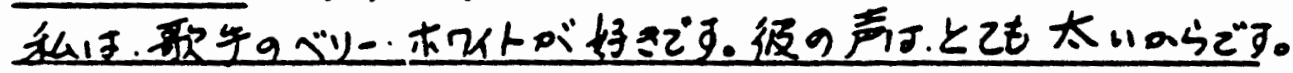

(24) I need these books, but they are too for me to buy. [A, DV]

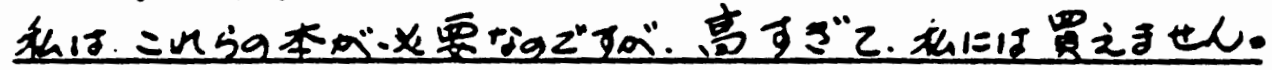

(25) That building is a library. It was built 150 years ago. [A, CV]

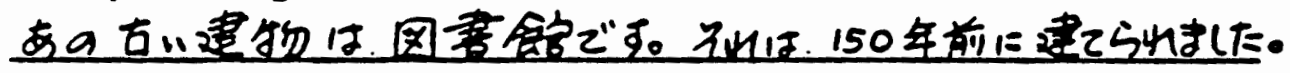

(26) My brother used to be very when he was a child. [A, CV]

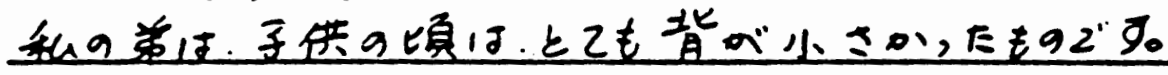

(27) Who is knocking on the door? What a knock it is! [A, DV]

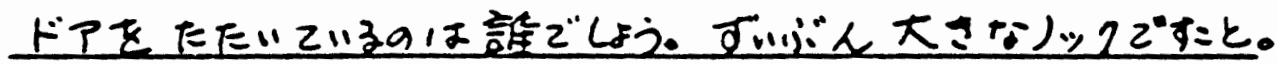

(28) This whiskey is too for me. May I have beer instead? [A, CG]

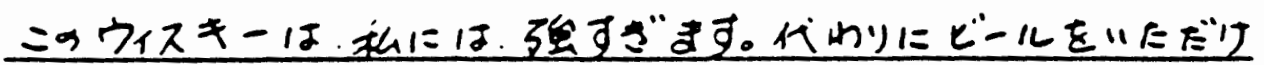

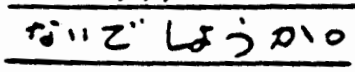


LEXICO-SEMANTICS TEST ANSWER SHEET

\section{VERBS}

(1) hear

(2) speak or talk

(3) draw

(4) drink

(5) watch

(6) have

(7) send

(8) take

(9) growing

(10) ride

(11) rent

(12) speak or talk

(13) eat

(14) boil

(15) drink

(16) had or dreamed

(17) hear

(18) wears

(19) cooked

(20) take

(21) boil

(22) send

(23) eat

(24) bring

(25) see

(26) cook

(27) take

(28) take

\section{ADJECTIVES}

(1) long

(2) thick or big

(3) green

(4) early

(5) small

(6) close

(7) deep

(8) thick

(9) high

(10) old

(11) small

(12) long

(13) Ioud

(14) cold

(15) deep

(16) quick

(17) short

(18) old

(19) strong

(20) blue

(21) strong

(22) cold

(23) deep

(24) expensive

(25) old

(26) short

(27) loud

(28) strong 


\section{APPENDIX B}

Portland State University TOEFI and Michigan test conversion chart:

TOEFL

Level 1: 400-425

Level 2: 426-450

Level 3: 451-480

Level 4: 481-525
MICHIGAN

$38-48$

49-64

$65-75$

$75-85$ 